Article

\title{
Grignard Reagent Utilization Enables a Practical and Scalable Construction of 3-Substituted 5-Chloro-1,6-naphthyridin-4-one Derivatives
}

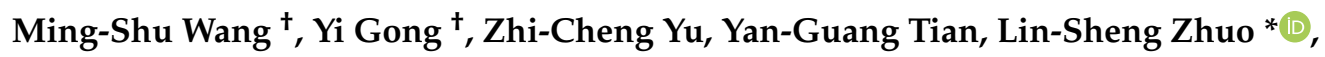 \\ Wei Huang *D and Neng-Fang She * \\ Key Laboratory of Pesticide \& Chemical Biology of Ministry of Education, International Joint Research Center \\ for Intelligent Biosensor Technology and Health, College of Chemistry, Central China Normal University, \\ Wuhan 430079, China; wms@mails.ccnu.edu.cn (M.-S.W.); gy@mails.ccnu.edu.cn (Y.G.); \\ yzc@mails.ccnu.edu.cn (Z.-C.Y.); tyguang1996@mails.ccnu.edu.cn (Y.-G.T.) \\ * Correspondence: zhuolinsheng199010@126.com (L.-S.Z.); weihuangwuhan@126.com (W.H.); \\ nfshe@mail.ccnu.edu.cn (N.-F.S.); Tel.: +86-27-67867706 (L.-S.Z. \& W.H.); +86-27-67867958 (N.-F.S.) \\ + These authors contributed equally to this work.
}

Received: 3 November 2020; Accepted: 27 November 2020; Published: 1 December 2020

\begin{abstract}
A robust, practical, and scalable approach for the construction of 3-substituted 5-chloro-1,6naphthyridin-4-one derivatives 13 via the addition of Grignard reagents to 4-amino-2-chloronicotinonitrile (15) was developed. Starting with various Grignard reagents, a wide range of 3-substituted 5-chloro-1,6-naphthyridin-4-one derivatives $\mathbf{1 3}$ were conveniently synthesized in moderate-to-good yields through addition-acidolysis-cyclocondensation. In addition, the robustness and applicability of this synthetic route was proven on a $100 \mathrm{~g}$ scale, which would enable convenient sample preparation in the preclinical development of 1,6-naphthyridin-4-one-based MET-targeting antitumor drug candidates.
\end{abstract}

Keywords: 1,6-naphthyridine; Grignard reagent; kinase inhibitor; antitumor drug candidate

\section{Introduction}

Naphthyridine scaffolds represent attractive building blocks widely used in pharmaceuticals [1,2], agrochemicals [3], and fluorescent probes [4-6]. Recently, it has been reported that numerous naphthyridine-containing molecules have promising medicinal properties for the prevention and treatment of angiogenic disorders [7], viral infections [8,9], bacterial infections [10-12], parasitic infections [13], heart failure [14], Alzheimer's disease (AD) [15], and cancers [16-24]. Therefore, the construction of naphthyridines has received increasing attention recently [25].

There are six naphthyridine isomers (1,5-, 1,6-, 1,7-, 1,8- 2,6-, and 2,7-pyridopyridines) [2]. Nowadays, the synthesis of 1,6-naphthyridine, especially for 1,6-naphthyridin-4-one derivatives, has attracted continuous interest in the pharmaceutical industry for its wide-ranging pharmacological activity [26,27], and it has been used as a multi-kinase inhibitor (I) [28], an anti-HCMV inhibitor (II) [29], an antidiabetic agent (III) [30], and antibacterial [31] and antiviral agents (IV) [1] (Figure 1).

In our previous report, 3-phenyl-1,6-naphthyridin-4-one was developed as a potent privileged skeleton for the discovery of a MET kinase inhibitor [20-23] from the key building block of 5-chloro-3-phenyl-1,6-naphthyridin-4-one (V). Extensive structure-activity relationship (SAR) studies and biological evaluations resulted in the discovery of two MET-targeting antitumor drug candidates, namely, VIa [20] and VIb [23], which are near the clinical stage (Figure 2). In addition to being used as MET kinase inhibitors, 3-phenyl-1,6-naphthyridin-4-one derivatives have also been reported as $\alpha 7$ nAChR allosteric modulators VII by ANVYL, LLC and its copartner (Figure 2) [32,33]. Thus, we propose that diversity-oriented synthesis (DOS), starting from 5-chloro-3-phenyl-1,6-naphthyridin-4-one (V), 
would resulted in more new drug lead compounds VIII based on the highly reactive 5-halo group and the functionalizable $\mathrm{NH}$ group.

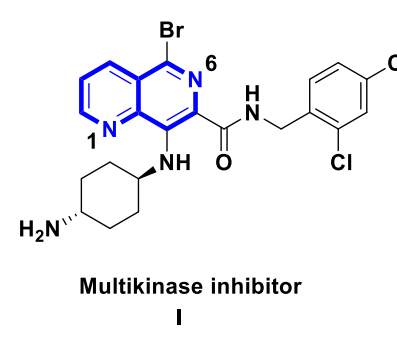

I

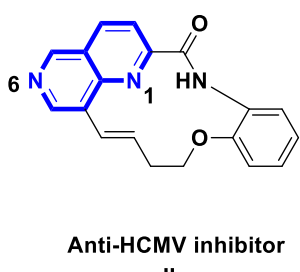

II

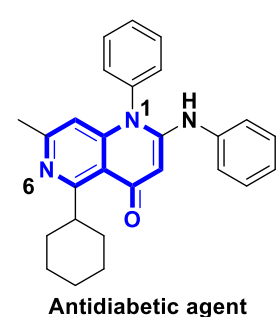

III

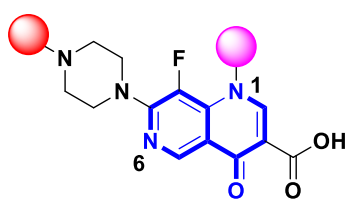

Antibacterial or antiviral agents IV

Figure 1. Representative 1,6-naphthyride- or 1,6-naphthyridin-4-one-based pharmacologically active compounds.

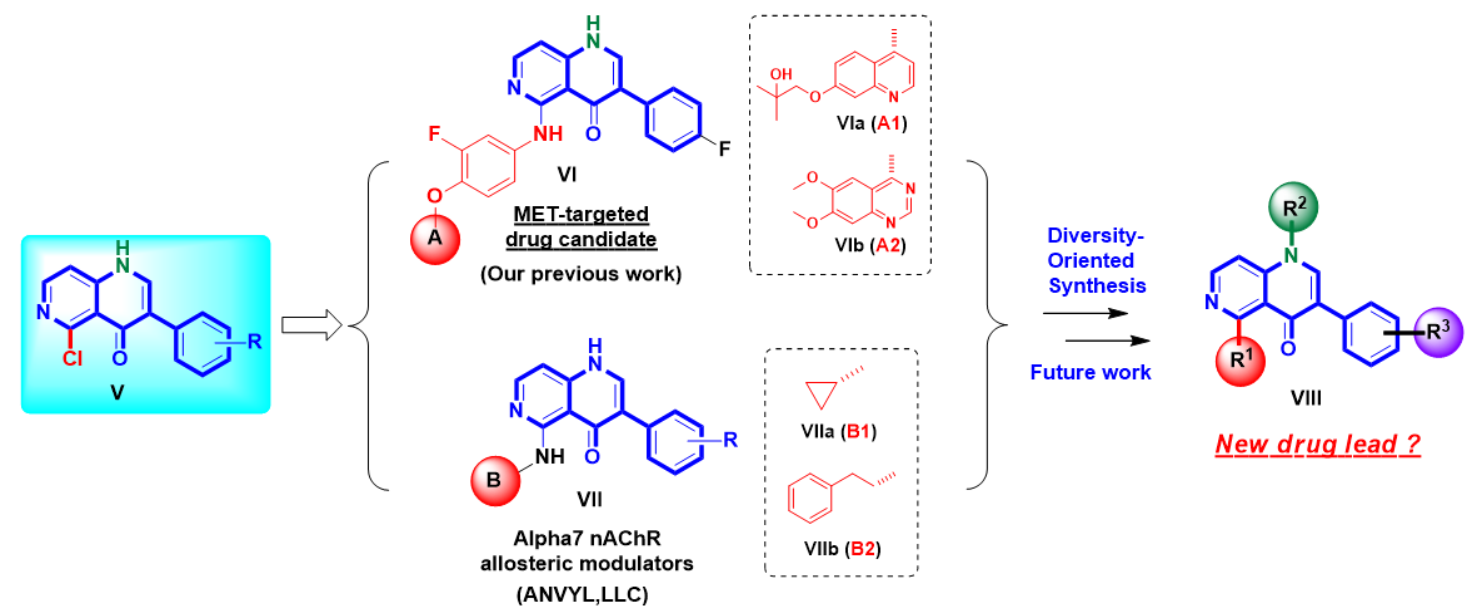

Figure 2. Discovery of bioactive 5-amino-3-phenyl-1,6-naphthyridin-4-one derivatives VI, VII and proposed future work.

However, the construction of the key 3-phenyl-1,6-naphthyridin-4-one skeleton has proven to be difficult. Currently, the reported procedures for its synthesis can be mainly divided into two types (Scheme 1a,b). The first method involves the preparation of an ethyl-2-(4,6-dichloro-nicotinoyl)-3(dimethylamino)acrylate intermediate (4), followed by strong base-catalyzed cyclization to generate the desired naphthyridine scaffold 5 . Then, the halodecarboxylation of intermediate 5 followed by Suzuki-Miyaura coupling with an arylboronic acid results in a 7-functionalized 1,6-naphthyridin-4-one 7 (Scheme 1a) [34]. Obviously, the synthetic route described above has inherent issues, including: (i) it is a labor-intensive process (five-step sequence); (ii) non-applicability on a large scale (due to the physical limitation of microwave heating, the penetration depth of only a few centimeters, and the limited dimensions of standing wave cavities) [35]; (iii) overfunctionalization of the $N$-position restricts its application in the drug discovery process for which $N$-substituents need to be finely regulated (the synthesis of the 1,6-naphthyridin-4-one scaffold, keeping a free $\mathrm{NH}$, using this synthetic route has not been reported and the introduction of $\mathrm{N}$-substituents in the initial step of the synthetic route makes the substituent regulation process very cumbersome) [36]. The second method was reported by ANVYL,LLC in their $\alpha 7 \mathrm{nAChR}$ allosteric modulators study [33], as well as our team in our MET kinase inhibitor study (Scheme 1b) [22]; knowledge of the mechanism of Conrad-Limpach cyclizations led to the introduction of a thermal cyclization to generate the desired naphthyridine scaffold. However, thermal cyclization is typically carried out in diphenyl ether at $230{ }^{\circ} \mathrm{C}$, and the lost control of chemoselectivity results in the generation of $\mathbf{1 3 a}$ and $\mathbf{1 4}$ in almost the same proportions. The high reaction temperature and the lack of chemoselectivity have resulted in the complex synthesis 
of product compositions and low yields (8.2\% for three steps), consequently leading to a labor-intensive enrichment and purification process.

Previous work:

a

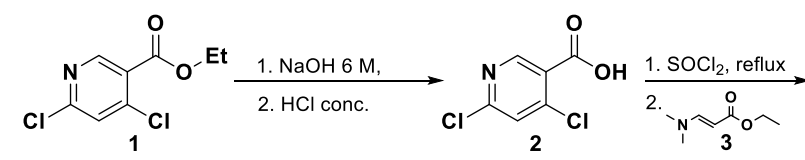<smiles>CCOC(=O)C(=CN(C)C)C(=O)c1cnc(Cl)cc1Cl</smiles>

1. Methylamine

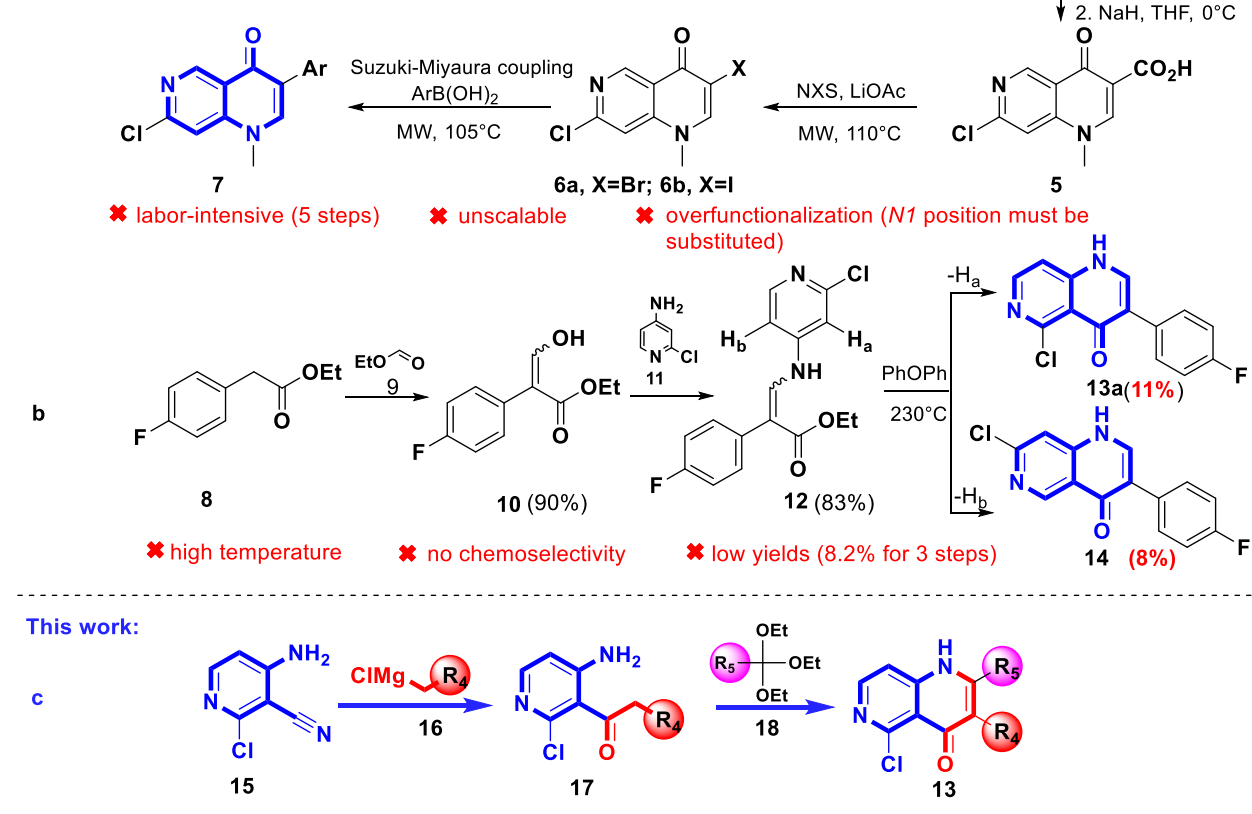

2. $\mathrm{NaH}, \mathrm{THF}, 0^{\circ} \mathrm{C}$

Scheme 1. Common construction strategies of the 3-phenyl-1,6-naphthyridin-4-one scaffold: (a) Suzuki-Miyaura coupling-based construction of 7-functionalized 1,6-naphthyridin-4-one 7; (b) Conrad-Limpach cyclizations-based construction of 5-chloro-3- substituted-1,6-naphthyridin-4-one derivatives 13a; (c) Grignard reagent-based strategy for the construction of diversified 5-chloro-3substituted-1,6-naphthyridin-4-one derivatives 13.

Altogether, the construction of 3-phenyl-1,6-naphthyridin-4-one derivatives is still a challenging subject. With our ongoing research interests in the discovery and development of 1,6-naphthyridin-4-one derivatives as drug candidates, we propose a facile Grignard reagent-based strategy through addition-acidolysis-cyclocondensation to provide diversified 5-chloro-3-substituted-1,6-naphthyridin-4-one derivatives 13 (Scheme 1c).

\section{Results and Discussion}

First, 5-chloro-3-(4-fluorophenyl)-1,6-naphthyridin-4-one (13a), which is the key common intermediate of our reported MET-targeting drug candidates, was chosen as a target molecule for the preliminary exploration of new synthetic routes. By retrosynthetic analysis, we reasoned that the cyclocondensation of 1-(4-amino-2-chloropyridin-3-yl)-2-(4-fluorophenyl)ethan-1-one (17a) and triethyl orthoformate would provide the desired molecule 13a (Scheme 2). Moreover, our way to intermediate $\mathbf{( 1 7} \mathbf{a})$ is based on the inclusion of a nitrile substituent at the 3-position of 4-amino-2-chloronicotinonitrile to enable the generation of an $\alpha$-keto group via Grignard chemistry. 


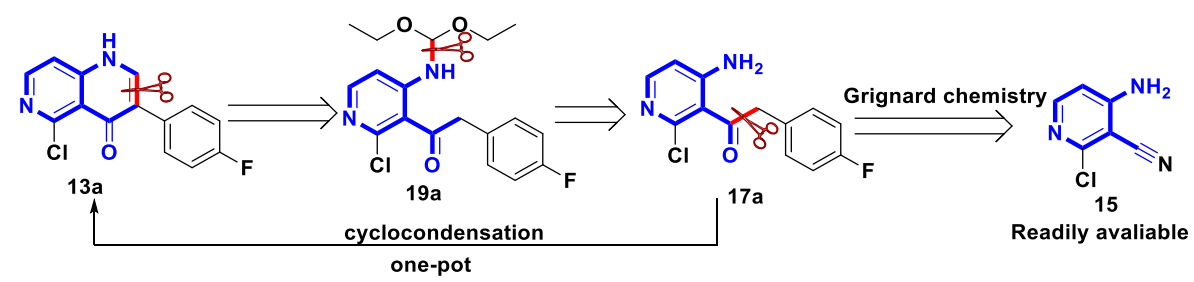

Scheme 2. Retrosynthetic analysis of 5-chloro-3-(4-fluorophenyl)-1,6-naphthyridin-4-one.

The overall synthetic route of 5-chloro-3-(4-fluorophenyl)-1,6-naphthyridin-4-one (13a) is shown in Scheme 3. The formation of 1-(4-amino-2-chloropyridin-3-yl)-2- (4-fluorophenyl)ethan-1-one (17a) was investigated first using 4-amino-2-chloronicotinonitrile (15) and 4-fluorobenzylmagnesium chloride (16a) (Scheme 4 and Table 1).

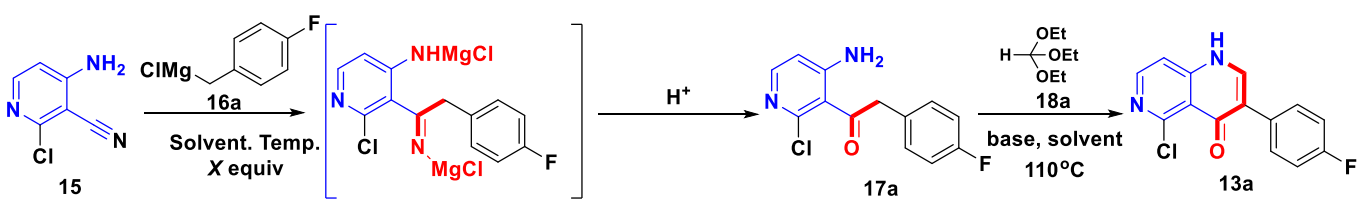

Scheme 3. An overall synthetic route of 5-chloro-3-(4-fluorophenyl)-1,6-naphthyridin-4-one.

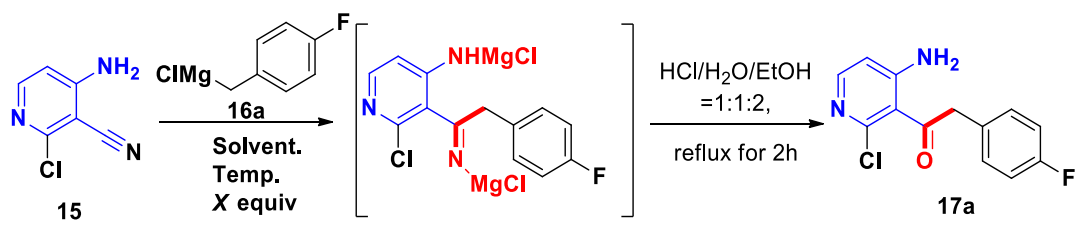

Scheme 4. Our planned route to key intermediate $\mathbf{1 7 a}$.

Table 1. Optimization of the reaction conditions ${ }^{\mathrm{a}, \mathrm{b}}$ for the synthesis of 1-(4-amino-2-chloropyridin-3-yl)2-(4-fluorophenyl)ethan-1-one (17a).

\begin{tabular}{|c|c|c|c|c|c|}
\hline Entry & 2-5 Equiv. & Solvent & Temp. $\left({ }^{\circ} \mathrm{C}\right)$ & Reaction Time (h) & Yield ${ }^{c}(\%)$ \\
\hline 1 & 2 & THF & 25 & 16 & 5 \\
\hline 2 & 2 & $\mathrm{Et}_{2} \mathrm{O}$ & 25 & 16 & 20 \\
\hline 3 & 2 & $\mathrm{Et}_{2} \mathrm{O}$ & 30 & 12 & 35 \\
\hline 4 & 2 & $\begin{array}{c}\text { Methyl } \\
\text { tert-butyl ether }\end{array}$ & 30 & 12 & 10 \\
\hline 5 & 2 & Isopropyl ether & 30 & 12 & 7 \\
\hline 6 & 2 & Toluene & 30 & 12 & Trace \\
\hline 7 & 3 & $\mathrm{Et}_{2} \mathrm{O}$ & 30 & 12 & 60 \\
\hline 8 & 4 & $\mathrm{Et}_{2} \mathrm{O}$ & 30 & 12 & 74 \\
\hline 9 & 5 & $\mathrm{Et}_{2} \mathrm{O}$ & 30 & 12 & 73 \\
\hline
\end{tabular}

${ }^{a}$ Reactions were performed using $0.5 \mathrm{mmol}$ of 4-amino-2-chloronicotinonitrile (15) in $1.0 \mathrm{~mL}$ solvent under $\mathrm{N}_{2}$ atmosphere. ${ }^{\mathrm{b}} 2 \mathrm{~mL} \mathrm{HCl} / \mathrm{H}_{2} \mathrm{O} / \mathrm{EtOH}$ (1:1:2) solvent was added for acidolysis. ${ }^{c}$ Isolated yield.

We initially subjected the two reactants to reaction at $25{ }^{\circ} \mathrm{C}$ for $16 \mathrm{~h}$ in two different solvents commonly used in Grignard chemistry, and it is obvious that diethyl ether exhibited a better effect on the reaction to provide product 17a than THF (Table 1, entries 1 and 2). It is also worth noting that the incomplete consumption of 4-amino-2-chloronicotinonitrile (15) was observed. Taking into consideration that lower temperatures can result in incomplete consumption and require longer reaction times, the reaction was warmed to $30{ }^{\circ} \mathrm{C}$. After $12 \mathrm{~h}$ of reaction, the consumption of 4-amino-2-chloronicotinonitrile (15) was no longer detected by HPLC dynamic detection, and the desired product $\mathbf{1 7}$ a was obtained at a $35 \%$ yield (Table 1, entry 3). Due to the low boiling point of ether, the temperature could not be increased further. Thus, different inert solvents with higher boiling points 
were evaluated as latent solvents in view of their versatility demonstrated in the Grignard reaction. Regrettably, lower yields were obtained if methyl tert-butyl ether (MTBE) and isopropyl ether (IPE) were utilized (Table 1, entries 4 and 5), and toluene did not favor the transformation either (Table 1 , entry 6). Finally, we optimized the reaction by changing the concentration of the Grignard reagent. As highlighted in Table 1 (entries 7-9), increasing the amount of Grignard reagent to 4.0 eqs afforded total consumption of 4-amino-2-chloronicotinonitrile (15) and resulted in a 74\% yield of the desired product (Table 1, entries 7 and 8). However, a further increase did not result in a further increase in yield (Table 1, entry 9), suggesting that 4.0 equiv. is the best amount. In summary, 1.0 equiv. of 4-amino-2-chloronicotinonitrile (15) and 4.0 equiv. of 4-fluorobenzylmagnesium chloride (16a) in ether at $30{ }^{\circ} \mathrm{C}$ for $12 \mathrm{~h}$ were determined to be the optimum reaction conditions.

With the optimized conditions established, the substrate scope with respect to the Grignard reagent was then evaluated (Scheme 5). It was found that the various Grignard reagents exposed to the optimized conditions smoothly provided the corresponding products $\mathbf{1 7 a}-\mathbf{n}$ in moderate to good yields (43-82\%). Typically, electron-withdrawing halogens on the benzyl Grignard reagent were well tolerated to provide the corresponding products in moderate-to-good yields $(\mathbf{1 7 a}, \mathbf{1 7 c}, \mathbf{1 7 e}$, and $\mathbf{1 7 f}$; $58-74 \%$ ); only the fluorine atom at the C-2 position of the benzyl group had a negative impact on the yield of the final products, which resulted in a relative lower yield of $\mathbf{1 7 b}$ and $\mathbf{1 7 d}$ with yields of $43 \%$ and 39\%, respectively. Notably, an electron-donating group (EDG) might be slightly better than an electron-withdrawing group (EWG), as exemplified by some typical EDGs, the alkyl-substituted benzyl Grignard reagents $\mathbf{1 7 h}-\mathbf{j}$ (75-80\%), including the bulky isopropyl group (17k; 71\%). Moreover, a naphthyl Grignard reagent and chain-growing alkyl Grignard reagents, such as phenethyl and ethyl Grignard reagents, could also provide good yields (17ln; 67-82\%).
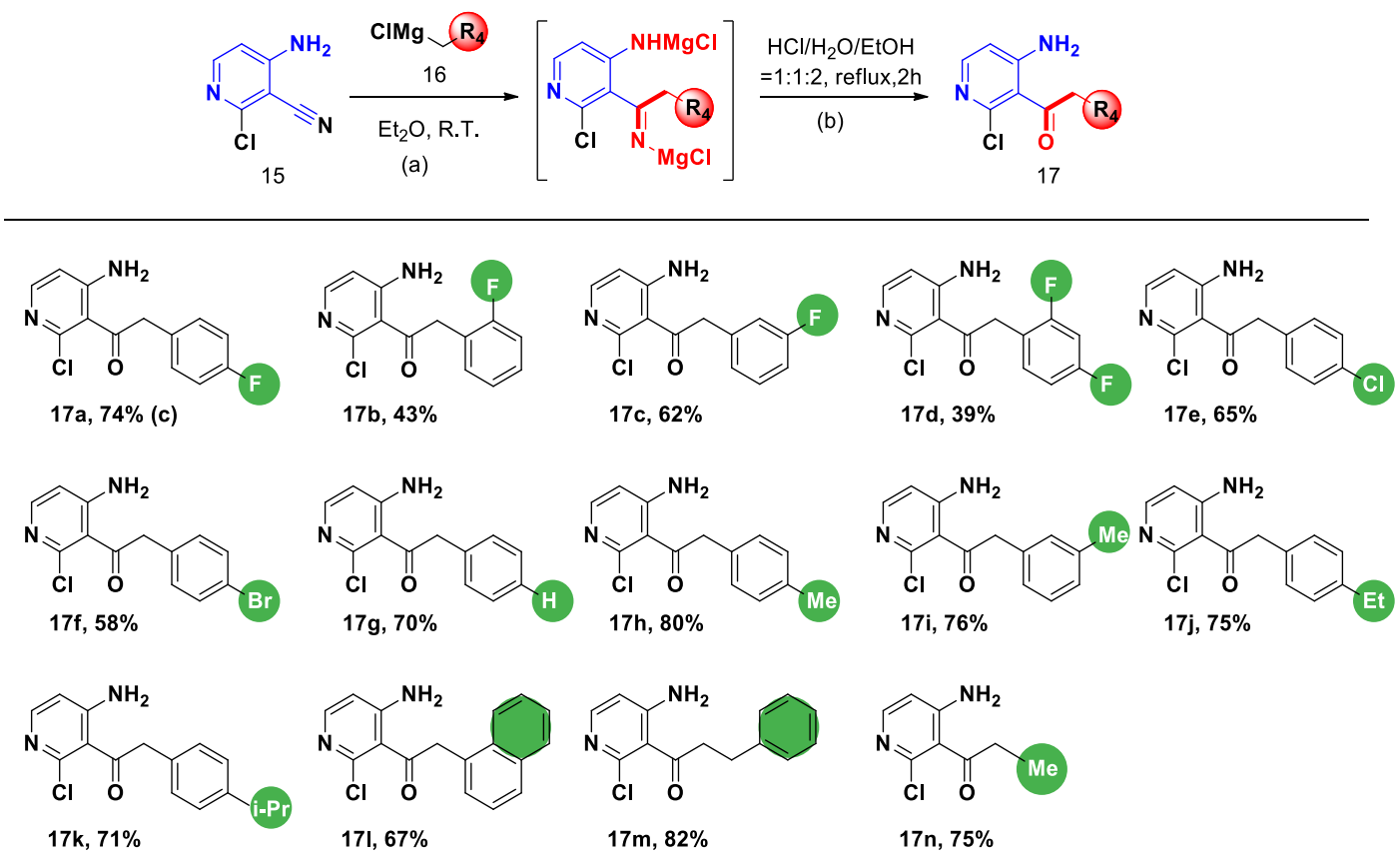

Scheme 5. Grignard reagent scope for the construction of 1, 6-naphthyridin-4-one scaffold. (a) 1.5 mmol of 4-amino-2-chloronicotinonitrile (15) and $6.0 \mathrm{mmol}$ of Grignard reagent (16) in $3.0 \mathrm{~mL}$ of ether at $30{ }^{\circ} \mathrm{C}$ for $12 \mathrm{~h}$ under an $\mathrm{N}_{2}$ atmosphere; (b) $6 \mathrm{~mL}$ of the $\mathrm{HCl} / \mathrm{H}_{2} \mathrm{O} / \mathrm{EtOH}$ (1:1:2) solvent was added for acidolysis; (c) Isolated yield.

With these positive results in hand, the optimal reaction conditions of the cyclocondensation of 1-(4-amino-2-chloropyridin-3-yl)-2-(4-fluorophenyl)ethan-1-one (17a) were investigated next (Scheme 6 and Table 2). Initially, we investigated cyclocondensation by employing $\mathrm{K}_{2} \mathrm{CO}_{3}$ as a base and DMF as a solvent at a temperature of $110{ }^{\circ} \mathrm{C}$. However, only a trace amount of the desired product was 
formed (Table 2, entry 1). Moreover, the combination of NaH and THF did not work (Table 2, entry 2). This was improved when we switched the inorganic base to an organic base. A 15\% yield of the desired product was observed when $\mathrm{Et}_{3} \mathrm{~N}$ was used (Table 2, entry 3), and a consistent improvement in the yield was observed by replacing $\mathrm{Et}_{3} \mathrm{~N}$ with DIPEA or DMAP (Table 2, entries 4 and 5), suggesting that DMAP is the best base. Then, by utilizing DMAP as the base, the solvent effects were also investigated. When triethoxymethane was used as both the reactant and solvent, the yield of the desired product was slightly increased (Table 2, entry 6). Finally, we turned our attention to base loading. By gradually reducing the amount of base, we found that only a catalytic amount of the base was needed (Table 2 , entries 6-9).
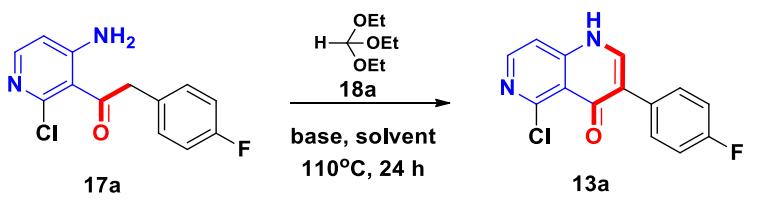

Scheme 6. Our planned route to target molecule 13a.

Table 2. Optimization of the reaction conditions ${ }^{\text {a }}$ for the cyclocondensation.

\begin{tabular}{cccccc}
\hline Entry & Base (equiv.) & Solvent & Temp. $\left({ }^{\circ} \mathbf{C}\right)$ & Reaction Time (h) & Yield $^{\mathbf{b}}(\mathbf{\%})$ \\
\hline 1 & $\mathrm{~K}_{2} \mathrm{CO}_{3}(2 \mathrm{eq})$ & $\mathrm{DMF}$ & 110 & 24 & $<1$ \\
2 & $\mathrm{NaH}(2 \mathrm{eq})$ & $\mathrm{THF}$ & 60 & 24 & $<1$ \\
3 & $\mathrm{Et}_{3} \mathrm{~N}(2 \mathrm{eq})$ & $\mathrm{DMF}$ & 110 & 24 & 15 \\
4 & $\mathrm{DIPEA}(2 \mathrm{eq})$ & $\mathrm{DMF}$ & 110 & 24 & 30 \\
5 & $\mathrm{DMAP}(2 \mathrm{eq})$ & $\mathrm{DMF}$ & 110 & 24 & 65 \\
6 & $\mathrm{DMAP}(2 \mathrm{eq})$ & $\mathrm{HC}(\mathrm{OEt})_{3}$ & 110 & 24 & 70 \\
7 & $\mathrm{DMAP}(1 \mathrm{eq})$ & $\mathrm{HC}(\mathrm{OEt})_{3}$ & 110 & 24 & 70 \\
8 & $\mathrm{DMAP}(0.1 \mathrm{eq})$ & $\mathrm{HC}(\mathrm{OEt})_{3}$ & 110 & 24 & 72 \\
9 & No catalyst & $\mathrm{HC}(\mathrm{OEt})_{3}$ & 110 & 24 & 0 \\
\hline
\end{tabular}

a Reactions were performed using $0.5 \mathrm{mmol}$ of 1-(4-amino-2-chloropyridin-3-yl)-2-(4-fluorophenyl)ethan-1-one (13a) in $1.0 \mathrm{~mL}$ of solvent under an air atmosphere. ${ }^{\mathrm{b}}$ Isolated yields.

Next, all of the intermediates synthesized above were subjected to the optimized reaction conditions. To our delight, the reaction demonstrated good compatibility, and a series of 3-substituted 1,6-naphthyridin-4-one derivatives were obtained smoothly with moderate-to-good yields (Scheme 7, 13a-o; 40-82\%). Generally, both 3-aryl-1,6-naphthyridin-4-one derivatives (13al; 40-82\%) and 3-alkyl-1,6-naphthyridin-4-one derivatives ( $\mathbf{1 3 m}$ and $\mathbf{1 3 n} ; 74 \%$ and $55 \%$ ) could be readily constructed, and the substituents on the benzene ring had less influence on the yields, except for an ortho-fluorine (13b and 13d; $46 \%$ and $40 \%$ ). Moreover, through changing the reactant triethyl orthoformate into triethyl orthoacetate, a methyl could be smoothly introduced to the C-2 position of the scaffold (13o, $69 \%$ ), which is of great benefit to the study of SARs in drug discovery. Taken together, this approach is a general method for building various 3-substituted 5-chloro-1,6-naphthyridin-4-one derivatives.

More importantly, the synthesis of compound 13a was performed on a $100 \mathrm{~g}$ scale to demonstrate the robustness and applicability of this synthetic route (Scheme 8). By starting from $153.0 \mathrm{~g}(1.0 \mathrm{~mol})$ of 4-amino-2-chloronicotinonitrile (15), we obtained the desired product at a yield of 53.3\% (total yield for two steps). After completing the enrichment of compound 13a, a kilogram- scale protocol for the creation of our 1,6-naphthyridin-4-one-based MET-targeting antitumor drug candidate VI was applied through acid-catalyzed nucleophilic substitution of 5-Cl atoms with high yields (90\%) and simple operation (insensitive to moisture and oxygen; filtration to obtain desired pure product). This would enable convenient sample preparation in the preclinical development of this drug candidate. 

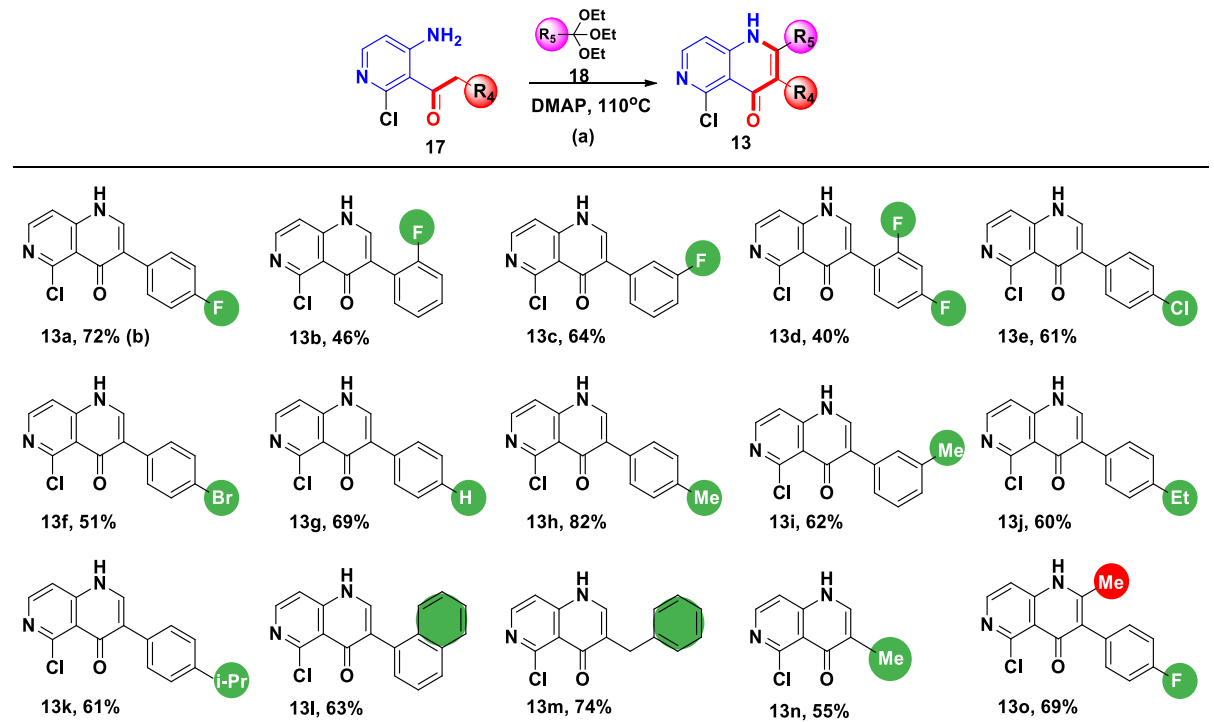

Scheme 7. Synthesis of 1,6-naphthyridin-4-one derivatives. (a) $0.5 \mathrm{mmol}$ of 1-(4-amino-2-chloropyridin3-yl)-2-substituted-ethan-1-one (17) and $0.05 \mathrm{mmol}$ of DMAP in $1.0 \mathrm{~mL}$ triethyl orthoformate (13a-n) or triethyl orthoacetate $(\mathbf{1 3 0})$ at $110{ }^{\circ} \mathrm{C}$ for $24 \mathrm{~h}$; (b) Isolated yield.

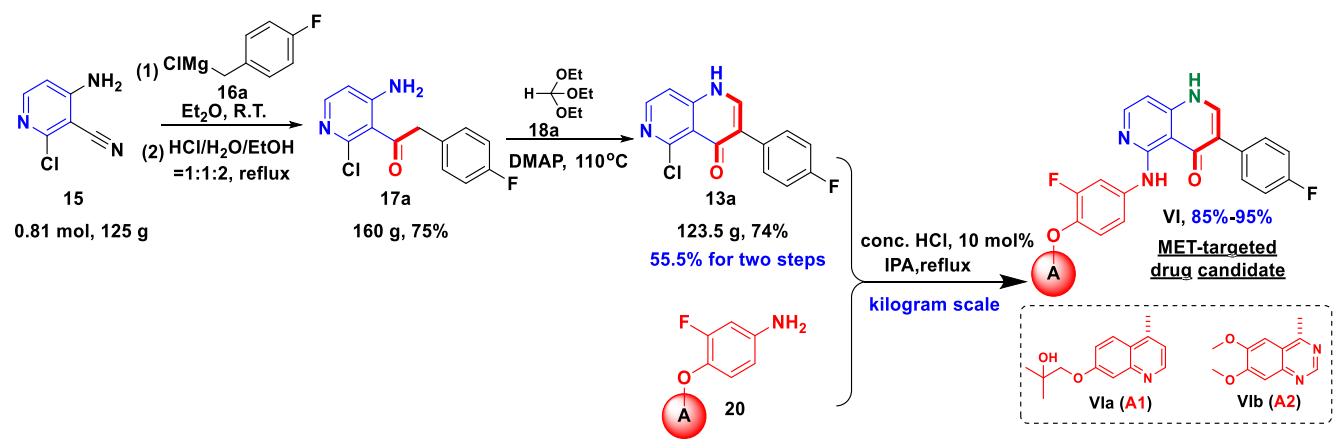

Scheme 8. The $100 \mathrm{~g}$ scale synthesis of 5-chloro-3-(4-fluorophenyl)-1,6-naphthyridin-4-one and its application in preclinical drug candidate enrichment.

\section{Conclusions}

In conclusion, we have developed a robust, practical, and scalable approach for the construction of 3-substituted 5-chloro-1,6-naphthyridin-4-one derivatives $\mathbf{1 3}$ via the addition of Grignard reagents to 4-amino-2-chloronicotinonitrile (15). Starting with various Grignard reagents through addition-acidolysis-cyclocondensation, a wide range of 5-chloro-3-substituted-1,6-naphthyridin-4-one derivatives 13 were synthesized in moderate-to-good yields. In addition, the robustness and applicability of this synthetic route was proven on a $100 \mathrm{~g}$ scale, which would enable convenient sample preparation in the preclinical development of 1,6-naphthyridin-4-one-based drug candidates. Further extensions of these naphthyridine derivatives in drug discovery are currently underway in our laboratories.

\section{Materials and Methods}

\subsection{General Information}

Unless otherwise noted, all chemical reagents were commercially available and treated with standard methods. Silica gel column chromatography (CC). silica gel (200-400 Mesh; Qingdao Makall Group Co., Ltd; Qingdao; China). Solvents were dried in a routine way and redistilled. All reactions 
involving air- or moisture-sensitive reagents were performed under a nitrogen or argon atmosphere. Melting points of compounds were measured on a Melt-Temp II apparatus (BUCHI Labortechnik AG, Uster, Switzerland) and are uncorrected. ${ }^{1} \mathrm{H}-\mathrm{NMR}$ spectra $(400 \mathrm{MHz})$ and ${ }^{13} \mathrm{C}-\mathrm{NMR}(100 \mathrm{MHz})$ spectra were recorded on an Ultrashield Plus AV 400 spectrometer (Bruker BioSpin AG, Fällanden, Switzerland) as dimethyl sulfoxide- $d_{6}$ (DMSO- $\left.d_{6}\right)$ solutions using tetramethylsilane (TMS) as an internal standard $(\delta=0)$ unless noted otherwise. ${ }^{1} \mathrm{H}-\mathrm{NMR}$ spectra $(600 \mathrm{MHz})$ and ${ }^{13} \mathrm{C}-\mathrm{NMR}(150 \mathrm{MHz})$ spectra were recorded on a Varian Mercury-Plus 600 spectrometer (Varian Inc., Palo Alto, CA, USA) as dimethyl sulfoxide- $d_{6}$ (DMSO- $\left.d_{6}\right)$ solutions using tetramethylsilane (TMS) as an internal standard $(\delta=0)$ unless noted otherwise, and all the ${ }^{1} \mathrm{H}$ and ${ }^{13} \mathrm{C}$ NMR Spectra are summarized in the Supplementary Materials. MS spectra were obtained on a 6120 quadrupole LC/MS (ESI, Agilent Technologies, Santa Clara, CA, USA). All reactions were monitored using thin-layer chromatography (TLC, Yantai Jiangyou Silica gel Development Co., Ltd.; Yantai; China) on silica gel plates. Yields were of purified compounds and were not optimized.

\subsection{General Procedure I for the Synthesis of 2-Substituted 1-(4-amino-2-chloropyridin-3-yl)-ethan-1-ones 17a-n}

The appropriate Grignard reagent 16 (6.0 mmol, 4.0 equivs.) in ether (3.3 mL) was added at room temperature to 4-amino-2-chloronicotinonitrile $(\mathbf{1 5}, 230.0 \mathrm{mg}, 1.5 \mathrm{mmol})$ in ether $(3.0 \mathrm{~mL})$. The reaction mixture was warmed to $30{ }^{\circ} \mathrm{C}$ and stirred for $12 \mathrm{~h}$ under ether atmosphere. Then the reaction mixture was cooled to $0{ }^{\circ} \mathrm{C}$ and $6.0 \mathrm{~mL}$ of mixed solvent $\left(\mathrm{HCl} / \mathrm{H}_{2} \mathrm{O} / \mathrm{EtOH}=1: 1: 2\right)$ was added dropwise, the reaction mixture was refluxed for a further $2 \mathrm{~h}$. After the completion of the reaction, the mixture was neutralized with sodium carbonate solution and extracted with EtOAc $(3 \times 15 \mathrm{~mL})$. The organic layer was dried with anhydrous sodium sulfate, filtered, and concentrated. The residue was purified by chromatography (hexane/EA $=5: 1$ ) to yield the corresponding compound 17 in good yield.

1-(4-Amino-2-chloropyridin-3-yl)-2-(4-fluorophenyl)ethan-1-one (17a) was prepared from 15 and (4-fluorobenzyl)magnesium chloride (16a) by general procedure I as a white solid (294 mg, 74\%), M.p. 130-133 ${ }^{\circ} \mathrm{C},{ }^{1} \mathrm{H}-\mathrm{NMR}\left(400 \mathrm{MHz}, \mathrm{DMSO}-d_{6}\right) \delta 7.81(\mathrm{~d}, J=6.0 \mathrm{~Hz}, 1 \mathrm{H}), 7.33-7.30(\mathrm{~m}, 2 \mathrm{H}), 7.17-7.12(\mathrm{~m}$, $2 \mathrm{H}), 6.61(\mathrm{~d}, J=6.0 \mathrm{~Hz}, 1 \mathrm{H}), 6.59(\mathrm{~s}, 2 \mathrm{H}), 4.18(\mathrm{~s}, 2 \mathrm{H}) .{ }^{13} \mathrm{C}-\mathrm{NMR}\left(100 \mathrm{MHz}, \mathrm{DMSO}-d_{6}\right) \delta 201.5,161.5(\mathrm{~d}$, $\left.J_{C-F}=241.0 \mathrm{~Hz}\right), 153.7,149.2,146.7,132.3\left(\mathrm{~d}, J_{C-F}=8.0 \mathrm{~Hz}\right), 130.5\left(\mathrm{~d}, J_{C-F}=3.0 \mathrm{~Hz}\right), 118.8,115.3\left(\mathrm{~d}, J_{C-F}\right.$ $=24.0 \mathrm{~Hz}), 110.5$, 49.1. MS (ESI): $265.05[\mathrm{M}+\mathrm{H}]^{+}$.

1-(4-Amino-2-chloropyridin-3-yl)-2-(2-fluorophenyl)ethan-1-one (17b) was prepared from $\mathbf{1 5}$ and (2-fluorobenzyl)magnesium chloride (16b) by general procedure I as a white solid (171 mg, 43\%), M.p. 127-131 ${ }^{\circ} \mathrm{C},{ }^{1} \mathrm{H}-\mathrm{NMR}\left(400 \mathrm{MHz}, \mathrm{DMSO}-d_{6}\right) \delta 7.82(\mathrm{~d}, J=6.0 \mathrm{~Hz}, 1 \mathrm{H}), 7.47-7.25(\mathrm{~m}, 2 \mathrm{H}), 7.24-7.07$ $(\mathrm{m}, 2 \mathrm{H}), 6.62(\mathrm{~s}, 2 \mathrm{H}), 6.60(\mathrm{~d}, J=6.0 \mathrm{~Hz}, 1 \mathrm{H}), 4.26(\mathrm{~s}, 2 \mathrm{H}) .{ }^{13} \mathrm{C}-\mathrm{NMR}\left(100 \mathrm{MHz}, \mathrm{DMSO}-d_{6}\right) \delta 200.2$, $162.2\left(\mathrm{~d}, J_{C-F}=243.0 \mathrm{~Hz}\right), 154.1,149.3,147.1,133.1\left(\mathrm{~d}, J_{C-F}=5.0 \mathrm{~Hz}\right), 129.6\left(\mathrm{~d}, J_{C-F}=8.0 \mathrm{~Hz}\right), 124.6$ $\left(\mathrm{d}, J_{C-F}=3.0 \mathrm{~Hz}\right), 121.7\left(\mathrm{~d}, J_{C-F}=16.0 \mathrm{~Hz}\right), 118.3,115.5\left(\mathrm{~d}, J_{C-F}=21.0 \mathrm{~Hz}\right), 110.7,43.8$. MS (ESI): $265.05[\mathrm{M}+\mathrm{H}]^{+}$.

1-(4-Amino-2-chloropyridin-3-yl)-2-(3-fluorophenyl)ethan-1-one (17c) was prepared from 15 and (3-fluorobenzyl)magnesium chloride (16c) by general procedure I as a white solid (246.3 mg, 62\%), M.p. 128-132 ${ }^{\circ} \mathrm{C},{ }^{1} \mathrm{H}-\mathrm{NMR}\left(400 \mathrm{MHz}, \mathrm{DMSO}-d_{6}\right) \delta 7.81(\mathrm{~d}, J=6.0 \mathrm{~Hz}, 1 \mathrm{H}), 7.43-7.29(\mathrm{~m}, 1 \mathrm{H}), 7.18-7.02$ $(\mathrm{m}, 3 \mathrm{H}), 6.62(\mathrm{~s}, 2 \mathrm{H}), 6.60(\mathrm{~d}, J=6.0 \mathrm{~Hz}, 1 \mathrm{H}), 4.23(\mathrm{~s}, 2 \mathrm{H}) .{ }^{13} \mathrm{C}-\mathrm{NMR}\left(100 \mathrm{MHz}, \mathrm{DMSO}-d_{6}\right) \delta 201.0$, $162.4\left(\mathrm{~d}, J_{C-F}=241.0 \mathrm{~Hz}\right), 153.8,149.2,146.7,137.1\left(\mathrm{~d}, J_{C-F}=8.0 \mathrm{~Hz}\right), 130.4\left(\mathrm{~d}, J_{C-F}=8.0 \mathrm{~Hz}\right), 126.6$ $\left(\mathrm{d}, J_{C-F}=2.0 \mathrm{~Hz}\right), 118.8,117.2\left(\mathrm{~d}, J_{C-F}=21.0 \mathrm{~Hz}\right), 114.1\left(\mathrm{~d}, J_{C-F}=21.0 \mathrm{~Hz}\right), 110.6,49.5$. MS (ESI): $265.05[\mathrm{M}+\mathrm{H}]^{+}$.

1-(4-Amino-2-chloropyridin-3-yl)-2-(2,4-difluorophenyl)ethan-1-one (17d) was prepared from $\mathbf{1 5}$ and (2,4-difluorobenzyl)magnesium chloride (16d) by general procedure I as a white solid (165.3 mg, 39\%), M.p. $180-183{ }^{\circ} \mathrm{C},{ }^{1} \mathrm{H}-\mathrm{NMR}\left(400 \mathrm{MHz}, \mathrm{DMSO}-d_{6}\right) \delta 7.83(\mathrm{~d}, J=6.0 \mathrm{~Hz}, 1 \mathrm{H}), 7.50-7.38(\mathrm{~m}, 1 \mathrm{H}), 7.26-7.15$ $(\mathrm{m}, 1 \mathrm{H}), 7.11-7.01(\mathrm{~m}, 1 \mathrm{H}), 6.62(\mathrm{~s}, 2 \mathrm{H}), 6.60(\mathrm{~d}, J=6.0 \mathrm{~Hz}, 1 \mathrm{H}), 4.25(\mathrm{~s}, 2 \mathrm{H}) .{ }^{13} \mathrm{C}-\mathrm{NMR}(100 \mathrm{MHz}$, DMSO- $\left.d_{6}\right) \delta 200.1,161.9\left(\mathrm{dd}, J_{C-F}=241.0 \mathrm{~Hz}, 12.0 \mathrm{~Hz}\right), 161.2\left(\mathrm{dd}, J_{C-F}=241.0 \mathrm{~Hz}, 12.0 \mathrm{~Hz}\right), 154.1$, $149.3,147.1,134.0\left(\mathrm{dd}, J_{C-F}=9.0 \mathrm{~Hz}, 6.0 \mathrm{~Hz}\right), 118.2,118.1\left(\mathrm{dd}, J_{C-F}=15.0 \mathrm{~Hz}, 3.0 \mathrm{~Hz}\right), 111.6\left(\mathrm{dd}, J_{C-F}\right.$ $=21.0 \mathrm{~Hz}, 5.0 \mathrm{~Hz}), 110.7,103.9\left(\mathrm{t}, J_{C-F}=26.0 \mathrm{~Hz}\right), 43.2 \mathrm{MS}(\mathrm{ESI}): 283.16[\mathrm{M}+\mathrm{H}]^{+}$. 
1-(4-Amino-2-chloropyridin-3-yl)-2-(4-chlorophenyl)ethan-1-one (17e) was prepared from 15 and (4-chlorobenzyl)magnesium chloride $(\mathbf{1 6 e})$ by general procedure I as a white solid $(273 \mathrm{mg}, 65 \%)$, M.p.147-150 ${ }^{\circ} \mathrm{C},{ }^{1} \mathrm{H}-\mathrm{NMR}\left(400 \mathrm{MHz}, \mathrm{DMSO}-\mathrm{d}_{6}\right) \delta 7.82(\mathrm{~d}, J=6.0 \mathrm{~Hz}, 1 \mathrm{H}), 7.38(\mathrm{~d}, J=8.4 \mathrm{~Hz}, 2 \mathrm{H}), 7.31$ $(\mathrm{d}, J=8.4 \mathrm{~Hz}, 2 \mathrm{H}), 6.62(\mathrm{~s}, 2 \mathrm{H}), 6.60(\mathrm{~d}, J=6.0 \mathrm{~Hz}, 1 \mathrm{H}), 4.19(\mathrm{~s}, 2 \mathrm{H}) .{ }^{13} \mathrm{C}-\mathrm{NMR}\left(100 \mathrm{MHz}, \mathrm{DMSO}-d_{6}\right) \delta$ 201.2, 153.7, 149.2, 146.7, 133.3, 132.2, 132.0, 128.5, 118.7, 110.5, 49.2. MS (ESI): 282.01 [M + H] ${ }^{+}$.

1-(4-Amino-2-chloropyridin-3-yl)-2-(4-bromophenyl)ethan-1-one (17f) was prepared from 15 and (4-bromobenzyl)magnesium chloride (16f) by general procedure I as a white solid (283.2 mg, 58\%), M.p. 132-133 ${ }^{\circ} \mathrm{C},{ }^{1} \mathrm{H}-\mathrm{NMR}\left(400 \mathrm{MHz}, \mathrm{DMSO}-d_{6}\right) \delta 7.82(\mathrm{~d}, J=6.0 \mathrm{~Hz}, 1 \mathrm{H}), 7.52(\mathrm{~d}, J=8.4 \mathrm{~Hz}, 2 \mathrm{H}), 7.25(\mathrm{~d}$, $J=8.4 \mathrm{~Hz}, 2 \mathrm{H}), 6.62(\mathrm{~s}, 2 \mathrm{H}), 6.60(\mathrm{~d}, J=6.0 \mathrm{~Hz}, 1 \mathrm{H}), 4.18(\mathrm{~s}, 2 \mathrm{H}) .{ }^{13} \mathrm{C}-\mathrm{NMR}\left(100 \mathrm{MHz}, \mathrm{DMSO}-d_{6}\right) \delta$ 201.1, 153.7, 149.2, 146.7, 133.8, 132.6, 131.4, 120.5, 118.7, 110.5, 49.3. MS (ESI): $326.96\left[\mathrm{M}+\mathrm{H}^{+}\right.$.

1-(4-Amino-2-chloropyridin-3-yl)-2-phenylethan-1-one (17g) was prepared from 15 and benzylmagnesium chloride (16g) by general procedure I as a white solid (258.9 $\mathrm{mg}, 70 \%)$, M.p.85-87 ${ }^{\circ} \mathrm{C}$, ${ }^{1} \mathrm{H}-\mathrm{NMR}\left(400 \mathrm{MHz}, \mathrm{DMSO}-d_{6}\right) \delta 7.81(\mathrm{~d}, J=6.0 \mathrm{~Hz}, 1 \mathrm{H}), 7.33-7.26(\mathrm{~m}, 5 \mathrm{H}), 6.62-6.59(\mathrm{~m}, 3 \mathrm{H}), 4.20(\mathrm{~s}$, $2 \mathrm{H}) .{ }^{13} \mathrm{C}-\mathrm{NMR}\left(100 \mathrm{MHz}, \mathrm{DMSO}-d_{6}\right) \delta 201.6,153.8,149.1,146.7,134.3,130.3,128.6,127.2,118.8,110.5$, 50.0. MS (ESI): $247.06[\mathrm{M}+\mathrm{H}]^{+}$.

1-(4-Amino-2-chloropyridin-3-yl)-2-(p-tolyl)ethan-1-one (17h) was prepared from $\mathbf{1 5}$ and (4-methylbenzyl)magnesium chloride (16h) by general procedure I as a white solid (312 $\mathrm{mg}, 80 \%)$, M.p. 100-103 ${ }^{\circ} \mathrm{C},{ }^{1} \mathrm{H}-\mathrm{NMR}\left(400 \mathrm{MHz}, \mathrm{DMSO}-d_{6}\right) \delta 7.80(\mathrm{~d}, J=6.0 \mathrm{~Hz}, 1 \mathrm{H}), 7.15(\mathrm{~d}, J=8.0 \mathrm{~Hz}, 2 \mathrm{H}), 7.11(\mathrm{~d}$, $J=8.0 \mathrm{~Hz}, 2 \mathrm{H}), 6.60(\mathrm{~d}, J=6.0 \mathrm{~Hz}, 3 \mathrm{H}), 4.13(\mathrm{~s}, 2 \mathrm{H}), 2.28(\mathrm{~s}, 3 \mathrm{H}) .{ }^{13} \mathrm{C}-\mathrm{NMR}\left(100 \mathrm{MHz}, \mathrm{DMSO}-d_{6}\right) \delta$ 201.7, 153.8, 148.9, 146.6, 136.2, 131.2, 129.2, 118.8, 110.5, 49.7, 21.1. MS (ESI): 261.32 [M + H] ${ }^{+}$.

1-(4-Amino-2-chloropyridin-3-yl)-2-(m-tolyl)ethan-1-one (17i) was prepared from $\mathbf{1 5}$ and (3-methylbenzyl)magnesium chloride (16i) by general procedure I as a white solid (297 mg, 76\%), M.p. 91-95 ${ }^{\circ} \mathrm{C}$, ${ }^{1} \mathrm{H}-\mathrm{NMR}\left(600 \mathrm{MHz}, \mathrm{DMSO}-d_{6}\right) \delta 7.80(\mathrm{~d}, J=6.0 \mathrm{~Hz}, 1 \mathrm{H}), 7.19(\mathrm{t}, J=7.2 \mathrm{~Hz}, 1 \mathrm{H}), 7.12-7.01(\mathrm{~m}, 3 \mathrm{H})$, 6.65-6.52 (m, 3H), $4.14(\mathrm{~s}, 2 \mathrm{H}), 2.28(\mathrm{~s}, 3 \mathrm{H}) .{ }^{13} \mathrm{C}-\mathrm{NMR}\left(150 \mathrm{MHz}, \mathrm{DMSO}-d_{6}\right) \delta 201.7,153.8,149.3,146.8$, $137.7,134.3,131.1,128.6,127.9,127.5,119.0,110.6,50.1,21.5$. MS (ESI): $261.08[\mathrm{M}+\mathrm{H}]^{+}$.

1-(4-Amino-2-chloropyridin-3-yl)-2-(4-ethylphenyl)ethan-1-one (17j) was prepared from 15 and (4-ethylbenzyl)magnesium chloride (16J) by general procedure I as a white solid (309 mg, 75\%), M.p. 89-102 ${ }^{\circ} \mathrm{C},{ }^{1} \mathrm{H}-\mathrm{NMR}\left(600 \mathrm{MHz}, \mathrm{DMSO}-d_{6}\right) \delta 7.80(\mathrm{~d}, J=6.0 \mathrm{~Hz}, 1 \mathrm{H}), 7.20-7.11(\mathrm{~m}, 4 \mathrm{H}), 6.60(\mathrm{~d}, J=6.0 \mathrm{~Hz}$, $3 \mathrm{H}), 4.14(\mathrm{~s}, 2 \mathrm{H}), 2.57(\mathrm{q}, J=7.2 \mathrm{~Hz}, 2 \mathrm{H}), 1.16(\mathrm{t}, J=7.2 \mathrm{~Hz}, 3 \mathrm{H}) .{ }^{13} \mathrm{C}-\mathrm{NMR}\left(150 \mathrm{MHz}, \mathrm{DMSO}-d_{6}\right) \delta 201.8$, 153.88, 149.1, 146.7, 142.7, 131.6, 130.3, 128.1, 119.0, 110.6, 49.7, 28.4, 16.2. MS (ESI): 275.09 [M + H] ${ }^{+}$.

1-(4-Amino-2-chloropyridin-3-yl)-2-(4-isopropylphenyl)ethan-1-one (17k) was prepared from $\mathbf{1 5}$ and (4-isopropylbenzyl)magnesium chloride (16k) by general procedure I as a white solid (307.5 mg, 71\%), M.p. 71-75 ${ }^{\circ} \mathrm{C},{ }^{1} \mathrm{H}-\mathrm{NMR}\left(600 \mathrm{MHz}, \mathrm{DMSO}-d_{6}\right) \delta 7.81(\mathrm{t}, J=5.2 \mathrm{~Hz}, 1 \mathrm{H}), 7.22-7.15(\mathrm{~m}, 4 \mathrm{H}), 6.62$ $(\mathrm{t}, J=4.4 \mathrm{~Hz}, 3 \mathrm{H}), 4.14(\mathrm{~s}, 1 \mathrm{H}), 2.85(\mathrm{p}, J=6.0 \mathrm{~Hz}, 1 \mathrm{H}), 1.27-1.14(\mathrm{~m}, 6 \mathrm{H}) .{ }^{13} \mathrm{C}-\mathrm{NMR}(150 \mathrm{MHz}$, DMSO- $\left.d_{6}\right) \delta 201.8,153.9,149.1,147.3,146.7,131.7,130.4,126.6,119.1,110.6,49.7,33.6,24.5$. MS (ESI): $289.11[\mathrm{M}+\mathrm{H}]^{+}$.

1-(4-Amino-2-chloropyridin-3-yl)-2-(naphthalen-1-yl)ethan-1-one (171) was prepared from 15 and (naphthalen-1-ylmethyl)magnesium chloride (161) by general procedure I as a white solid (297.6 mg, 67\%), M.p. 78-80 ${ }^{\circ} \mathrm{C},{ }^{1} \mathrm{H}-\mathrm{NMR}\left(400 \mathrm{MHz}, \mathrm{DMSO}-d_{6}\right) \delta 7.87-7.86(\mathrm{~m}, 1 \mathrm{H}), 7.85-7.84(\mathrm{~m}, 1 \mathrm{H}), 7.83-7.81$ $(\mathrm{m}, 2 \mathrm{H}), 7.53-7.50(\mathrm{~m}, 2 \mathrm{H}), 7.48-7.46(\mathrm{~m}, 2 \mathrm{H}), 6.66(\mathrm{~s}, 2 \mathrm{H}), 6.65(\mathrm{~d}, J=6.0 \mathrm{~Hz}, 1 \mathrm{H}), 4.70(\mathrm{~s}, 2 \mathrm{H}) .{ }^{13} \mathrm{C}-\mathrm{NMR}$ $\left(100 \mathrm{MHz}, \mathrm{DMSO}-d_{6}\right) \delta 201.3,154.2,149.0,147.1,133.7,132.6,131.1,129.1,128.8,128.0,126.5,126.1$, 125.9, 124.8, 118.6, 110.8, 47.7. MS (ESI): $297.07[\mathrm{M}+\mathrm{H}]^{+}$.

1-(4-Amino-2-chloropyridin-3-yl)-3-phenylpropan-1-one (17m) was prepared from $\mathbf{1 5}$ and phenethylmagnesium chloride (16m) by general procedure I as a white solid (320.7 mg, 82\%), M.p. 81-83 ${ }^{\circ} \mathrm{C}$, ${ }^{1} \mathrm{H}-\mathrm{NMR}\left(400 \mathrm{MHz}, \mathrm{DMSO}-\mathrm{d}_{6}\right) \delta 7.80(\mathrm{~d}, J=6.0 \mathrm{~Hz}, 1 \mathrm{H}), 7.41-7.08(\mathrm{~m}, 5 \mathrm{H}), 6.61(\mathrm{~d}, J=6.0 \mathrm{~Hz}, 1 \mathrm{H})$, $6.58(\mathrm{~s}, 2 \mathrm{H}), 3.15(\mathrm{t}, J=7.6 \mathrm{~Hz}, 2 \mathrm{H}), 2.94(\mathrm{t}, J=7.6 \mathrm{~Hz}, 2 \mathrm{H}) .{ }^{13} \mathrm{C}-\mathrm{NMR}\left(100 \mathrm{MHz}, \mathrm{DMSO}-d_{6}\right) \delta 203.2$, 153.8, 149.1, 146.8, 141.1, 128.8, 128.7, 126.4, 118.8, 110.5, 45.3, 29.8.MS (ESI): 261.91 [M + H] $]^{+}$.

1-(4-Amino-2-chloropyridin-3-yl)propan-1-one (17n) was prepared from 15 and ethylmagnesium chloride (16n) by general procedure I as a white solid (210 mg, 75\%), M.p. 97-100 ${ }^{\circ} \mathrm{C},{ }^{1} \mathrm{H}-\mathrm{NMR}$ $\left(400 \mathrm{MHz}, \mathrm{DMSO}-\mathrm{d}_{6}\right) \delta 7.80(\mathrm{~d}, J=6.0 \mathrm{~Hz}, 1 \mathrm{H}), 6.61(\mathrm{~d}, J=6.0 \mathrm{~Hz}, 1 \mathrm{H}), 6.50(\mathrm{~s}, 2 \mathrm{H}), 2.82(\mathrm{q}, J=$ 
$7.2 \mathrm{~Hz}, 2 \mathrm{H}), 1.09(\mathrm{t}, J=7.2 \mathrm{~Hz}, 3 \mathrm{H}) .{ }^{13} \mathrm{C}-\mathrm{NMR}\left(100 \mathrm{MHz}, \mathrm{DMSO}-d_{6}\right) \delta 204.7,153.5,149.0,146.5,119.3$, 110.4, 37.1, 8.3. MS (ESI): $185.04[\mathrm{M}+\mathrm{H}]^{+}$.

\subsection{General Procedure II for the Synthesis of 3-Substituted 1,6-naphthyridin-4-ones 13a-o}

A solution of 2-substituted 1-(4-amino-2-chloropyridin-3-yl)-ethan-1-one 17 (0.5 mmol) and DMAP $(0.5 \mathbf{~ m m o l})$ in triethyl orthoformate $(\mathbf{1 8 a}$, for $\mathbf{1 3 a}-\mathbf{n})$ or triethyl orthoacetate $(\mathbf{1 8 b}$, for $\mathbf{1 3 0})(1.0 \mathrm{~mL})$ was heated to $110{ }^{\circ} \mathrm{C}$ and stirred for $24 \mathrm{~h}$, then cooled to room temperature, the resulted solid was filtered and the filter residue was added to a $5 \mathrm{~mL}$ round-bottomed flask charged with $2.0 \mathrm{~mL}$ isopropanol, refluxed for $2 \mathrm{~h}$ and filtered immediately to afford the corresponding compound $\mathbf{1 3}$ in good yield.

5-Chloro-3-(4-fluorophenyl)-1,6-naphthyridin-4-one (13a) was prepared from 17a and triethyl orthoformate (18a) by general procedure II as a light yellow solid (98 mg, 72\%), M.p. 296-299 ${ }^{\circ} \mathrm{C}$, ${ }^{1} \mathrm{H}-\mathrm{NMR}\left(400 \mathrm{MHz}, \mathrm{DMSO}-d_{6}\right) \delta 12.3(\mathrm{~s}, 1 \mathrm{H}), 8.31(\mathrm{~d}, J=5.6 \mathrm{~Hz}, 1 \mathrm{H}), 8.15(\mathrm{~s}, 1 \mathrm{H}), 7.69-7.65(\mathrm{~m}, 2 \mathrm{H})$, $7.45(\mathrm{~d}, J=5.6 \mathrm{~Hz}, 1 \mathrm{H}), 7.25-7.20(\mathrm{~m}, 2 \mathrm{H}) .{ }^{13} \mathrm{C}-\mathrm{NMR}\left(100 \mathrm{MHz}, \mathrm{DMSO}-d_{6}\right) \delta 173.8,161.8\left(\mathrm{~d}, J_{\mathrm{C}-F}=\right.$ $243.0 \mathrm{~Hz}), 150.6,148.2,147.7,138.2,131.8\left(\mathrm{~d}, J_{C-F}=3.0 \mathrm{~Hz}\right), 131.1\left(\mathrm{~d}, J_{C-F}=7.0 \mathrm{~Hz}\right), 124.5,118.0,115.1$ $\left(\mathrm{d}, J_{\mathrm{C}-F}=21.0 \mathrm{~Hz}\right), 113.1$. MS (ESI): $275.21[\mathrm{M}+\mathrm{H}]^{+}$.

5-Chloro-3-(2-fluorophenyl)-1,6-naphthyridin-4(1H)-one (13b) was prepared from $\mathbf{1 7 b}$ and triethyl orthoformate (18a) by general procedure II as a light yellow solid (63 mg, 46\%), M.p. $264-268{ }^{\circ} \mathrm{C}$, ${ }^{1} \mathrm{H}-\mathrm{NMR}\left(400 \mathrm{MHz}, \mathrm{DMSO}-d_{6}\right) \delta 12.38(\mathrm{~s}, 1 \mathrm{H}), 8.33(\mathrm{~d}, J=6.0 \mathrm{~Hz}, 1 \mathrm{H}), 8.11(\mathrm{~s}, 1 \mathrm{H}), 7.54-7.34(\mathrm{~m}, 3 \mathrm{H})$, 7.32-7.14 (m, 2H). ${ }^{13} \mathrm{C}-\mathrm{NMR}\left(150 \mathrm{MHz}, \mathrm{DMSO}-d_{6}\right) \delta 173.2,160.7\left(\mathrm{~d}, J_{\mathrm{C}-F}=244.5 \mathrm{~Hz}\right), 150.4,148.4$, $147.9,139.2,132.6,129.9\left(\mathrm{~d}, J_{C-F}=7.5 \mathrm{~Hz}\right), 124.4,123.3\left(\mathrm{~d}, J_{C-F}=13.5 \mathrm{~Hz}\right), 121.3,117.6,115.7\left(\mathrm{~d}, J_{C-F}=\right.$ $22.5 \mathrm{~Hz}), 113.1$. MS (ESI): $275.23[\mathrm{M}+\mathrm{H}]^{+}$.

5-Chloro-3-(3-fluorophenyl)-1,6-naphthyridin-4(1H)-one (13c) was prepared from $\mathbf{1 7 c}$ and triethyl orthoformate (18a) by general procedure II as a light yellow solid $(87.9 \mathrm{mg}, 64 \%)$, M.p. $273-275{ }^{\circ} \mathrm{C}$, ${ }^{1} \mathrm{H}-\mathrm{NMR}\left(400 \mathrm{MHz}\right.$, DMSO- $\left.d_{6}\right) \delta 12.41(\mathrm{~s}, 1 \mathrm{H}), 8.33(\mathrm{~d}, J=6.0 \mathrm{~Hz}, 1 \mathrm{H}), 8.25(\mathrm{~s}, 1 \mathrm{H}), 7.59-7.38(\mathrm{~m}, 4 \mathrm{H})$, 7.20-7.10 (m, 1H). ${ }^{13} \mathrm{C}-\mathrm{NMR}\left(150 \mathrm{MHz}, \mathrm{DMSO}-d_{6}\right) \delta 173.7,162.3\left(\mathrm{~d}, J_{\mathrm{C}-F}=244.5 \mathrm{~Hz}\right), 150.7,148.4$, $147.7\left(\mathrm{~d}, J_{C-F}=4.5 \mathrm{~Hz}\right), 138.9,137.9,130.2,125.0,123.9\left(\mathrm{~d}, J_{C-F}=4.5 \mathrm{~Hz}\right), 118.1\left(\mathrm{~d}, J_{C-F}=4.5 \mathrm{~Hz}\right)$, $115.8\left(\mathrm{~d}, J_{C-F}=22.5 \mathrm{~Hz}\right), 114.2\left(\mathrm{~d}, J_{C-F}=13.5 \mathrm{~Hz}\right), 113.15, \mathrm{MS}(\mathrm{ESI}): 275.03[\mathrm{M}+\mathrm{H}]^{+}$.

5-Chloro-3-(2,4-difluorophenyl)-1,6-naphthyridin-4(1H)-one (13d) was prepared from $\mathbf{1 7 d}$ and triethyl orthoformate (18a) by general procedure II as a light yellow solid (58.5 mg, 40\%), M.p. 238-241 ${ }^{\circ} \mathrm{C}$, ${ }^{1} \mathrm{H}-\mathrm{NMR}\left(400 \mathrm{MHz}\right.$, DMSO- $\left.d_{6}\right) \delta 12.58(\mathrm{~s}, 1 \mathrm{H}), 8.89(\mathrm{~s}, 1 \mathrm{H}), 7.93(\mathrm{~d}, J=6.0 \mathrm{~Hz}, 1 \mathrm{H}), 7.68-7.62(\mathrm{~m}, 1 \mathrm{H})$, $7.46\left(\mathrm{td}, J_{1}=9.6 \mathrm{~Hz}, J_{2}=2.4 \mathrm{~Hz}, 1 \mathrm{H}\right), 7.28\left(\mathrm{td}, J_{1}=8.4 \mathrm{~Hz}, J_{2}=2.4 \mathrm{~Hz}, 1 \mathrm{H}\right), 7.05(\mathrm{~d}, J=6.0 \mathrm{~Hz}$, $1 \mathrm{H}) .{ }^{13} \mathrm{C}-\mathrm{NMR}\left(100 \mathrm{MHz}, \mathrm{DMSO}-d_{6}\right) \delta 171.6,165.9\left(\mathrm{dd}, J_{\mathrm{C}-F}=298.0 \mathrm{~Hz}, 12.0 \mathrm{~Hz}\right), 163.0\left(\mathrm{dd}, J_{\mathrm{C}-F}=\right.$ $298.0 \mathrm{~Hz}, 12.0 \mathrm{~Hz}), 160.6,149.3,148.2,139.0,133.8\left(\mathrm{dd}, J_{\mathrm{C}-F}=10.0 \mathrm{~Hz}, 4.0 \mathrm{~Hz}\right), 116.5,116.1\left(\mathrm{dd}, J_{C-F}=\right.$ $15.0 \mathrm{~Hz}, 4.0 \mathrm{~Hz}), 112.4,\left(\mathrm{dd}, J_{C-F}=21.0 \mathrm{~Hz}, 4.0 \mathrm{~Hz}\right), 109.1,104.9\left(\mathrm{t}, J_{C-F}=21.0 \mathrm{~Hz}\right), 101.7$. MS (ESI): $293.42[\mathrm{M}+\mathrm{H}]^{+}$.

5-Chloro-3-(4-chlorophenyl)-1,6-naphthyridin-4(1H)-one (13e) was prepared from $\mathbf{1 7 e}$ and triethyl orthoformate (18a) by general procedure II as a light yellow solid $(88.5 \mathrm{mg}, 61 \%)$, M.p. $196-200{ }^{\circ} \mathrm{C}$, ${ }^{1} \mathrm{H}-\mathrm{NMR}\left(400 \mathrm{MHz}\right.$, DMSO- $\left.d_{6}\right) \delta 12.60(\mathrm{~s}, 1 \mathrm{H}), 8.32(\mathrm{~d}, J=6.0 \mathrm{~Hz}, 1 \mathrm{H}), 8.19(\mathrm{~s}, 1 \mathrm{H}), 7.68(\mathrm{~d}, J=8.4 \mathrm{~Hz}$, $2 \mathrm{H}), 7.49(\mathrm{~d}, J=6.0 \mathrm{~Hz}, 1 \mathrm{H}), 7.45(\mathrm{~d}, J=8.4 \mathrm{~Hz}, 2 \mathrm{H}) .{ }^{13} \mathrm{C}-\mathrm{NMR}\left(100 \mathrm{MHz}, \mathrm{DMSO}-d_{6}\right) \delta 173.7,150.6$, 148.2, 147.6, 138.4, 134.3, 132.0, 130.8, 128.3, 124.0, 118.0, 113.1. MS (ESI): $292.11[\mathrm{M}+\mathrm{H}]^{+}$.

3-(4-Bromophenyl)-5-chloro-1,6-naphthyridin-4(1H)-one (13f) was prepared from $\mathbf{1 7 f}$ and triethyl ortho- formate (18a) by general procedure II as a light yellow solid $(85.6 \mathrm{mg}, 51 \%)$, M.p. $239-241{ }^{\circ} \mathrm{C}$, ${ }^{1} \mathrm{H}-\mathrm{NMR}\left(400 \mathrm{MHz}\right.$, DMSO- $\left.d_{6}\right) \delta 12.36(\mathrm{~s}, 1 \mathrm{H}), 8.32(\mathrm{~d}, J=6.0 \mathrm{~Hz}, 1 \mathrm{H}), 8.18(\mathrm{~s}, 1 \mathrm{H}), 7.64-7.58(\mathrm{~m}, 4 \mathrm{H})$, $7.45(\mathrm{~d}, J=6.0 \mathrm{~Hz}, 1 \mathrm{H}) .{ }^{13} \mathrm{C}-\mathrm{NMR}\left(100 \mathrm{M}, \mathrm{DMSO}_{-} d_{6}\right) \delta 173.6,150.6,148.2,147.6,138.3,134.7,131.2$, 131.1, 124.1, 120.6, 118.0, 113.0. MS (ESI): $336.95[\mathrm{M}+\mathrm{H}]^{+}$.

5-Chloro-3-phenyl-1,6-naphthyridin-4(1H)-one (13g) was prepared from $\mathbf{1 7} \mathbf{g}$ and triethyl orthoformate (18a) by general procedure II as a light yellow solid (89.8 mg, 69\%), M.p. $198-200{ }^{\circ} \mathrm{C},{ }^{1} \mathrm{H}-\mathrm{NMR}$ $\left(400 \mathrm{MHz}\right.$, DMSO- $\left.d_{6}\right) \delta 12.22(\mathrm{~s}, 1 \mathrm{H}), 8.21(\mathrm{~d}, J=6.0 \mathrm{~Hz}, 1 \mathrm{H}), 8.03(\mathrm{~d}, J=5.6 \mathrm{~Hz}, 1 \mathrm{H}), 7.53(\mathrm{~d}, J=$ $1.6 \mathrm{~Hz}, 1 \mathrm{H}), 7.52(\mathrm{~s}, 1 \mathrm{H}), 7.38-7.18(\mathrm{~m}, 4 \mathrm{H}) .{ }^{13} \mathrm{C}-\mathrm{NMR}\left(100 \mathrm{MHz}, \mathrm{DMSO}-d_{6}\right) \delta 173.9,150.7,148.1,147.7$, $138.1,135.5,129.2,128.3,127.5,125.6,118.0,113.0$. MS (ESI): $292.11[\mathrm{M}+\mathrm{H}]^{+}$. 
5-Chloro-3-(p-tolyl)-1,6-naphthyridin-4(1H)-one (13h) was prepared from $\mathbf{1 7 h}$ and triethyl orthoformate (18a) by general procedure II as a light yellow solid (110.9 mg, 82\%), M.p. $222-225{ }^{\circ} \mathrm{C},{ }^{1} \mathrm{H}-\mathrm{NMR}$ $\left(400 \mathrm{MHz}, \mathrm{DMSO}-\mathrm{d}_{6}\right) \delta 12.28(\mathrm{~s}, 1 \mathrm{H}), 8.30(\mathrm{~d}, J=6.0 \mathrm{~Hz}, 1 \mathrm{H}), 8.09(\mathrm{~s}, 1 \mathrm{H}), 7.53(\mathrm{~d}, J=8.4 \mathrm{~Hz}, 2 \mathrm{H}), 7.46$ $(\mathrm{d}, J=6.0 \mathrm{~Hz}, 1 \mathrm{H}), 7.20(\mathrm{~d}, J=8.4 \mathrm{~Hz}, 2 \mathrm{H}), 2.33(\mathrm{~s}, 3 \mathrm{H}) .{ }^{13} \mathrm{C}-\mathrm{NMR}\left(100 \mathrm{MHz}, \mathrm{DMSO}-d_{6}\right) \delta 173.9,150.6$, 148.0, 147.5, 137.7, 136.6, 132.5, 128.9, 128.8, 125.4, 117.9, 113.0, 21.2. MS (ESI): 271.85[M + H] ${ }^{+}$.

5-Chloro-3-(m-tolyl)-1,6-naphthyridin-4(1H)-one (13i) was prepared from $\mathbf{1 7} \mathbf{i}$ and triethyl orthoformate (18a) by general procedure II as a light yellow solid (83.9 mg, 62\%), M.p. $257-261{ }^{\circ} \mathrm{C}$, ${ }^{1} \mathrm{H}-\mathrm{NMR}\left(600 \mathrm{MHz}, \mathrm{DMSO}-d_{6}\right) \delta 12.32(\mathrm{~s}, 1 \mathrm{H}), 8.31(\mathrm{~d}, J=6.0 \mathrm{~Hz}, 1 \mathrm{H}), 8.12(\mathrm{~s}, 1 \mathrm{H}), 7.46-7.44(\mathrm{~m}$, $2 \mathrm{H}), 7.40(\mathrm{~d}, J=6.0 \mathrm{~Hz}, 1 \mathrm{H}), 7.30(\mathrm{t}, J=7.6 \mathrm{~Hz}, 1 \mathrm{H}), 7.14(\mathrm{~d}, J=7.6 \mathrm{~Hz}, 1 \mathrm{H}), 2.35(\mathrm{~s}, 3 \mathrm{H}) .{ }^{13} \mathrm{C}-\mathrm{NMR}$ $\left(150 \mathrm{MHz}, \mathrm{DMSO}-d_{6}\right) \delta 173.9,150.7,148.2,147.7,138.1,137.3,135.5,129.8,128.3,128.2,126.3,125.7$, 118.0, 113.1, 21.7. MS (ESI): $271.85[\mathrm{M}+\mathrm{H}]^{+}$.

5-Chloro-3-(4-ethylphenyl)-1,6-naphthyridin-4(1H)-one (13j) was prepared from $\mathbf{1 7 J}$ and triethyl ortho- formate (18a) by general procedure II as a light yellow solid (85.4 mg, 60\%), M.p. $270-273{ }^{\circ} \mathrm{C}$, ${ }^{1} \mathrm{H}-\mathrm{NMR}\left(600 \mathrm{MHz}, \mathrm{DMSO}-d_{6}\right) \delta 12.30(\mathrm{~s}, 1 \mathrm{H}), 8.30(\mathrm{~d}, J=6.0 \mathrm{~Hz}, 1 \mathrm{H}), 8.11(\mathrm{~s}, 1 \mathrm{H}), 7.57-7.49(\mathrm{~m}, 2 \mathrm{H})$, $7.45(\mathrm{~d}, J=6.0 \mathrm{~Hz}, 1 \mathrm{H}), 7.23(\mathrm{~d}, J=7.2 \mathrm{~Hz}, 2 \mathrm{H}), 2.62(\mathrm{q}, J=7.2 \mathrm{~Hz}, 2 \mathrm{H}), 1.20(\mathrm{t}, J=7.2 \mathrm{~Hz}, 3 \mathrm{H})$. ${ }^{13}$ C-NMR (150 MHz, DMSO- $\left.d_{6}\right) \delta 174.0,150.7,148.2,147.7,143.2,137.9,132.9,129.2,127.9,125.6,118.0$, 113.1, 28.5, 16.3. MS (ESI): 285.65[M + H] ${ }^{+}$.

5-Chloro-3-(4-isopropylphenyl)-1,6-naphthyridin-4(1H)-one (13k) was prepared from 17k and triethyl orthoformate (18a) by general procedure II as a light yellow solid (91.1 mg, 61\%), M.p. 303-304 ${ }^{\circ} \mathrm{C}$, ${ }^{1} \mathrm{H}-\mathrm{NMR}\left(600 \mathrm{MHz}, \mathrm{DMSO}-\mathrm{d}_{6}\right) 12.39(\mathrm{~s}, 1 \mathrm{H}), 8.42(\mathrm{~d}, J=6.0 \mathrm{~Hz}, 1 \mathrm{H}), 8.24(\mathrm{~s}, 1 \mathrm{H}), 7.60(\mathrm{~d}, J=6.0 \mathrm{~Hz}$, $1 \mathrm{H}), 7.55(\mathrm{~d}, J=8.4 \mathrm{~Hz}, 2 \mathrm{H}), 7.28(\mathrm{~d}, J=8.4 \mathrm{~Hz}, 2 \mathrm{H}), 2.99-2.86(\mathrm{~m}, 1 \mathrm{H}), 1.23(\mathrm{~d}, J=7.2 \mathrm{~Hz}, 6 \mathrm{H})$. ${ }^{13}$ C-NMR (150 MHz, DMSO-d 6 ) $\delta 174.0,150.7,148.1,147.8,147.7,137.9,133.1,129.2,126.3,125.7,118.0$, 113.1, 33.8, 24.5. MS (ESI): 299.63[M + H] $]^{+}$.

5-Chloro-3-(naphthalen-1-yl)-1,6-naphthyridin-4(1H)-one (131) was prepared from $\mathbf{1 7 1}$ and triethyl orthoformate (18a) by general procedure II as a light yellow solid (96.6 mg, 63\%), M.p. $281-283{ }^{\circ} \mathrm{C}$, ${ }^{1} \mathrm{H}-\mathrm{NMR}\left(400 \mathrm{MHz}, \mathrm{DMSO}-d_{6}\right) \delta 12.36(\mathrm{~s}, 1 \mathrm{H}), 8.37(\mathrm{~d}, J=5.6 \mathrm{~Hz}, 1 \mathrm{H}), 8.12-8.07(\mathrm{~m}, 1 \mathrm{H}), 8.01-7.91(\mathrm{~m}$, $2 \mathrm{H}), 7.71-7.63(\mathrm{~m}, 1 \mathrm{H}), 7.62-7.49(\mathrm{~m}, 3 \mathrm{H}), 7.49-7.37(\mathrm{~m}, 2 \mathrm{H}) .{ }^{13} \mathrm{C}-\mathrm{NMR}\left(100 \mathrm{MHz}, \mathrm{DMSO}-d_{6}\right) \delta 174.3$, $150.6,148.3,148.2,139.2,134.2,133.6,132.5,128.5,128.4,128.3,126.6,126.3,126.2,125.9,117.8,113.2$. MS (ESI): 307.91[M + H] $]^{+}$.

3-Benzyl-5-chloro-1,6-naphthyridin-4(1H)-one $(\mathbf{1 3 m})$ was prepared from $\mathbf{1 7 m}$ and triethyl orthoformate (18a) by general procedure II as a light yellow solid (100 mg, 74\%), M.p. $212-215{ }^{\circ} \mathrm{C}$, ${ }^{1} \mathrm{H}-\mathrm{NMR}\left(400 \mathrm{MHz}, \mathrm{DMSO}-\mathrm{d}_{6}\right) \delta 12.00(\mathrm{~s}, 1 \mathrm{H}), 8.26(\mathrm{~d}, J=6.0 \mathrm{~Hz}, 1 \mathrm{H}), 7.89(\mathrm{~s}, 1 \mathrm{H}), 7.39(\mathrm{~d}, J=6.0 \mathrm{~Hz}$, 1H), 7.34-7.21 (m, 4H), 7.21-7.09 (m, 1H), 3.73 (s, 2H). ${ }^{13} \mathrm{C}-\mathrm{NMR}\left(100 \mathrm{MHz}, \mathrm{DMSO}-d_{6}\right) \delta 174.7,150.3$, $147.8,140.9,137.3,129.0,128.6,126.3,126.2,116.9,113.4,33.5$. MS (ESI): $271.77[\mathrm{M}+\mathrm{H}]^{+}$.

5-Chloro-3-methyl-1,6-naphthyridin-4(1H)-one (13n) was prepared from $\mathbf{1 7 n}$ and triethyl orthoformate (18a) by general procedure II as a light yellow solid (53.3 mg, 55\%), M.p. 181-185 ${ }^{\circ} \mathrm{C}$, ${ }^{1} \mathrm{H}-\mathrm{NMR}\left(400 \mathrm{MHz}, \mathrm{DMSO}-\mathrm{d}_{6}\right) \delta 11.94(\mathrm{~s}, 1 \mathrm{H}), 8.23(\mathrm{~d}, J=6.0 \mathrm{~Hz}, 1 \mathrm{H}), 7.89(\mathrm{~s}, 1 \mathrm{H}), 7.36(\mathrm{~d}, J=6.0 \mathrm{~Hz}$, 1H), 1.93 (s, 3H). ${ }^{13} \mathrm{C}-\mathrm{NMR}\left(100 \mathrm{MHz}, \mathrm{DMSO}-d_{6}\right) \delta 175.6,150.2,147.8,147.5,136.4,122.7,116.3,112.9$, 14.1. MS (ESI): $285.69[\mathrm{M}+\mathrm{H}]^{+}$.

5-Chloro-3-(4-fluorophenyl)-2-methyl-1,6-naphthyridin-4(1H)-one (13o) was prepared from $\mathbf{1 7 0}$ and triethyl orthoacetate $(\mathbf{1 8 b})$ by general procedure II as a light yellow solid (99.4 mg, 69\%), M.p. 309-311 ${ }^{\circ} \mathrm{C},{ }^{1} \mathrm{H}-\mathrm{NMR}\left(600 \mathrm{MHz}, \mathrm{DMSO}-d_{6}\right) \delta 12.02(\mathrm{~s}, 1 \mathrm{H}), 8.28(\mathrm{~d}, J=6.0 \mathrm{~Hz}, 1 \mathrm{H}), 7.40(\mathrm{~d}, J=6.0 \mathrm{~Hz}$, $1 \mathrm{H}), 7.30-6.98(\mathrm{~m}, 4 \mathrm{H}), 2.16(\mathrm{~s}, 3 \mathrm{H}) .{ }^{13} \mathrm{C}-\mathrm{NMR}\left(150 \mathrm{MHz}, \mathrm{DMSO}-d_{6}\right) \delta 173.9,161.8\left(\mathrm{~d}, J_{\mathrm{C}-F}=241.0 \mathrm{~Hz}\right)$, $150.5,148.3,147.6,147.1,133.2\left(\mathrm{~d}, J_{C-F}=7.5 \mathrm{~Hz}\right), 131.8\left(\mathrm{~d}, J_{C-F}=1.5 \mathrm{~Hz}\right), 124.9,116.7,115.3\left(\mathrm{~d}, J_{C-F}=\right.$ 21.0 Hz), 112.6, 19.1. MS (ESI): $289.51[\mathrm{M}+\mathrm{H}]^{+}$. 


\subsection{Scale-Up Experiment}

\subsubsection{Mass Preparation of 1-(4-Amino-2-chloropyridin-3-yl)-2-(4-fluorophenyl)ethan-1-one (17a)}

To a 5 L four-neck flask 4-amino-2-chloronicotinonitrile 15 (125.0 g, $0.81 \mathrm{~mol})$ and ether $(1500 \mathrm{~mL})$ were added, followed by (4-fluorobenzyl)magnesium chloride in ether (16a) (1.8 M, $3.24 \mathrm{~mol}, 1800 \mathrm{~mL}$, 4.0 equivs.) at room temperature. The mixture was warmed to $30{ }^{\circ} \mathrm{C}$ and stirred for $12 \mathrm{~h}$ under ether atmosphere. Then the reaction was cooled in an ice-water bath with the reaction flask equipped with a distillation unit, and $3000 \mathrm{~mL}$ of mixed solvent $\left(\mathrm{HCl} / \mathrm{H}_{2} \mathrm{O} / \mathrm{EtOH}=1: 1: 2\right)$ was added dropwise, the volatilized ether was recovered with a distillation unit, and the reaction mixture was refluxed for a further $2 \mathrm{~h}$. After the completion of the reaction, ethanol was removed under vacuum, and the residue was stirred for $12 \mathrm{~h}$ and filtered to afford $199 \mathrm{~g}$ of crude hydrochloride of $17 \mathrm{a}$ as a yellow solid. Next, the yellow solid was added to a $2.0 \mathrm{~L}$ flask charged with ethanol $(720 \mathrm{~mL})$ and warmed up to reflux followed by addition of trimethylamine $(0.66 \mathrm{~mol}, 66.86 \mathrm{~g})$ dropwise. The resulted mixture was refluxed for further $1 \mathrm{~h}$, then cooled to room temperature and stirred overnight. Finally, filtered and the filter cake was washed with 50\% ethanol in water to afford 17a as a light yellow solid, (160 g, 75\%).

\subsubsection{Mass Preparation of 5-Chloro-3-(4-fluorophenyl)-1,6-naphthyridin-4-one (13a)}

To a $2.0 \mathrm{~L}$ of four-neck flask equipped with a distillation unit was added 1-(4-amino-2chloropyridin-3-yl)-2-(4-fluorophenyl)ethan-1-one (17a, $0.604 \mathrm{~mol}, 160.0 \mathrm{~g})$ and triethyl orthoformate (18a, $1600 \mathrm{~mL})$, the reaction mixture was warmed up to $100{ }^{\circ} \mathrm{C}$ and stirred for $1.5 \mathrm{~h}$, then DMAP $(0.1 \mathrm{eq}$, $0.6 \mathrm{~mol}, 7.3 \mathrm{~g}$ ) was added and the stirring was continued for $24 \mathrm{~h}$ at $100{ }^{\circ} \mathrm{C}$ with the produced ethanol collected by distillation unit. After the completion of the reaction, the mixture was cooled to room temperature and filtered, the filter cake was added to a $2.0 \mathrm{~L}$ of four-neck flask charged with $1500 \mathrm{~mL}$ isopropanol, refluxed for $5 \mathrm{~h}$ and filtered immediately to afford 13a as light yellow solid $(123.5 \mathrm{~g}, 74)$.

Supplementary Materials: The supplementary materials are available online.

Author Contributions: W.H. and N.-F.S. conceived and designed the experiments; M.W., Y.G., Y.-G.T. and L.-S.Z. performed synthesis; M.-S.W. and L.-S.Z. analyzed the data; M.-S.W., Z.-C.Y. and W.H. wrote the paper. W.H. was responsible for the correspondence of the manuscript. All authors have read and agreed to the published version of the manuscript.

Funding: This work was supported by the National Key Research and Development Program of China (No. 2017YFA0505200), the Key Research and Development Program of Hubei Province, China (2020BCB042), the Science and Technology Program of Wuhan (No. 2019020701011460). Dedicated to the 100th anniversary of Chemistry at Nankai University.

Conflicts of Interest: The authors declare no conflict of interest.

\section{References}

1. Tabarrini, O.; Massari, S.; Sancineto, L.; Daelemans, D.; Sabatini, S.; Manfroni, G.; Cecchetti, V.; Pannecouque, C. Structural Investigation of the Naphthyridone Scaffold: Identification of a 1,6-Naphthyridone Derivative with Potent and Selective Anti-HIV Activity. ChemMedChem 2011, 6, 1249-1257. [CrossRef] [PubMed]

2. Madaan, A.; Verma, R.; Kumar, V.; Singh, A.T.; Jain, S.K.; Jaggi, M. 1,8-Naphthyridine Derivatives: A Review of Multiple Biological Activities. Arch. Pharm. 2015, 348, 837-860. [CrossRef] [PubMed]

3. Martínez-Teipel, B.; Teixidó, J.; Pascual, R.; Mora, M.; Pujolà, J.; Fujimoto, T.; Borrell, J.I.; Michelotti, E.L. 2-Methoxy-6-oxo-1,4,5,6-tetrahydropyridine-3-carbonitriles: Versatile Starting Materials for the Synthesis of Libraries with Diverse Heterocyclic Scaffolds. J. Comb. Chem. 2005, 7, 436-448. [CrossRef] [PubMed]

4. Zhang, Y.; Sun, R.; Kang, X.; Wang, D.-H.; Chen, Y. A water-soluble 1,8-naphthyridine-based imidazolium molecular gripper for fluorescence sensing and discriminating of GMP. Dye. Pigment. 2020, 174, 108103. [CrossRef]

5. Ren, R.; Xu, H.-C.; Dong, H.; Peng, H.-T.; Wu, P.-P.; Qiu, Y.; Yang, S.-G.; Sun, Q.; She, N.-F. Ultrafast 2,7-Naphthyridine-Based fluorescent probe for detection of thiophenol with a remarkable Stokes shift and its application In vitro and in vivo. Talanta 2019, 205, 120067. [CrossRef] 
6. Sun, Q.; Ren, R.; Wu, P.-P.; Zhuo, L.-S.; Dong, H.; Peng, H.-T.; Cao, Y.-F.; Luo, X.-G.; She, N.-F. A 2, 7-naphthyridine-based fluorescent turn-on probe for detection of biothiols in vitro and in vivo. Dye. Pigment. 2020, 182, 108702. [CrossRef]

7. Mu, X.-Y.; Xu, J.; Zhou, Y.-J.; Li, Y.-L.; Liu, Y.; Wang, X.-S. Convenient synthesis of naphtho[1,6] naphthyridine derivatives under catalyst-free conditions. Res. Chem. Intermed. 2013, 41, 1703-1714. [CrossRef]

8. Singh, I.P.; Kumar, S.; Gupta, S. Naphthyridines with Antiviral Activity-A Review. Med. Chem. 2017, 13, 430-438. [CrossRef]

9. Peese, K.M.; Allard, C.W.; Connolly, T.; Johnson, B.L.; Li, C.; Patel, M.; Sorensen, M.E.; Walker, M.A.; Meanwell, N.A.; McAuliffe, B.; et al. 5,6,7,8-Tetrahydro-1,6-naphthyridine Derivatives as Potent HIV-1-Integrase-Allosteric-Site Inhibitors. J. Med. Chem. 2019, 62, 1348-1361. [CrossRef]

10. De Morais Oliveira-Tintino, C.D.; Tintino, S.R.; Muniz, D.F.; dos Santos Barbosa, C.R.; Pereira, R.L.S.; Begnini, I.M.; Rebelo, R.A.; da Silva, L.E.; Mireski, S.L.; Nasato, M.C. Do 1,8-naphthyridine sulfonamides possess an inhibitory action against TetK and MrsA efflux pumps in multiresistant Staphylococcus aureus strains? Microb. Pathog. 2020, 147, 104268. [CrossRef]

11. Yoo, B.K.; Triller, D.M.; Yong, C.-S.; Lodise, T.P. Gemifloxacin: A New Fluoroquinolone Approved for Treatment of Respiratory Infections. Ann. Pharmacother. 2004, 38, 1226-1235. [CrossRef] [PubMed]

12. Hong, C.Y.; Kim, Y.K.; Chang, J.H.; Kim, S.H.; Choi, H.; Nam, D.H.; Kim, Y.Z.; Kwak, J.H. Novel Fluoroquinolone Antibacterial Agents Containing Oxime-Substituted (Aminomethyl) pyrrolidines: Synthesis and Antibacterial Activity of 7-(4-(Aminomethyl)-3-(methoxyimino) pyrrolidin-1-yl)1-cyclopropyl-6-fluoro-4-oxo-1,4-dihydro[1,8] naphthyridine-3-carboxylic Acid (LB20304). J. Med. Chem. 1997, 40, 3584-3593.

13. Thomas, M.; De Rycker, M.; Wall, R.J.; Spinks, D.; Epemolu, O.; Manthri, S.; Norval, S.; Osuna-Cabello, M.; Patterson, S.; Riley, J. Identification and optimisation of a series of 8-hydroxy naphthyridines with potent in vitro antileishmanial activity: Initial SAR and assessment of in vivo activity. J. Med. Chem. 2020, 63, 9523-9539. [CrossRef]

14. Meredith, E.L.; Ardayfio, O.; Beattie, K.; Dobler, M.R.; Enyedy, I.; Gaul, C.; Hosagrahara, V.; Jewell, C.; Koch, K.; Lee, W.; et al. Identification of Orally Available Naphthyridine Protein Kinase D Inhibitors. J. Med. Chem. 2010, 53, 5400-5421. [CrossRef] [PubMed]

15. Fiorito, J.; Vendome, J.; Saeed, F.; Staniszewski, A.; Zhang, H.; Yan, S.; Deng, S.-X.; Arancio, O.; Landry, D.W. Identification of a Novel 1,2,3,4-Tetrahydrobenzo[b][1,6]naphthyridine Analogue as a Potent Phosphodiesterase 5 Inhibitor with Improved Aqueous Solubility for the Treatment of Alzheimer's Disease. J. Med. Chem. 2017, 60, 8858-8875. [CrossRef] [PubMed]

16. Srivastava, S.K.; Jha, A.; Agarwal, S.K.; Mukherjee, R.; Burman, A.C. Synthesis and structure-activity relationships of potent antitumor active quinoline and naphthyridine derivatives. Anti Cancer Agents Med. Chem. 2007, 7, 685-709. [CrossRef]

17. Zhuo, L.-S.; Xu, H.; Wang, M.-S.; Zhao, X.-E.; Ming, Z.-H.; Zhu, X.-L.; Huang, W.; Yang, G. 2,7-naphthyridinone-based MET kinase inhibitors: A promising novel scaffold for antitumor drug development. Eur. J. Med. Chem. 2019, 178, 705-714. [CrossRef]

18. Wang, M.-S.; Xu, H.; Gong, Y.; Qu, R.-Y.; Zhuo, L.-S.; Huang, W. Efficient Arylation of 2,7-Naphthyridin-1(2H)-one with Diaryliodonium Salts and Discovery of a New Selective MET/AXL Kinase Inhibitor. ACS Comb. Sci. 2020, 22, 9, 457-467. [CrossRef]

19. Sun, H.; Zhuo, L.; Dong, H.; Huang, W.; She, N.-F. Discovery of 8-Amino-Substituted 2-Phenyl-2,7-Naphthyridinone Derivatives as New c-Kit/VEGFR-2 Kinase Inhibitors. Molecules 2019, 24, 4461. [CrossRef]

20. Chen, T.; Zhuo, L.-S.; Liu, P.-F.; Fang, W.-R.; Li, Y.; Huang, W. Discovery of 1,6-naphthyridinone-based MET kinase inhibitor bearing quinoline moiety as promising antitumor drug candidate. Eur. J. Med. Chem. 2020, 192, 112174. [CrossRef]

21. Xu, H.; Wang, M.; Wu, F.; Zhuo, L.; Huang, W.; She, N.-F. Discovery of N-substituted-3-phenyl1,6-naphthyridinone derivatives bearing quinoline moiety as selective type II c-Met kinase inhibitors against VEGFR-2. Bioorganic Med. Chem. 2020, 28, 115555. [CrossRef] [PubMed]

22. Wang, M.-S.; Zhuo, L.-S.; Yang, F.-P.; Wang, W.-J.; Huang, W.; Yang, G.-F. Synthesis and biological evaluation of new MET inhibitors with 1,6-naphthyridinone scaffold. Eur. J. Med. Chem. 2019, 185, 111803. [CrossRef] [PubMed] 
23. Wu, F.; Zhuo, L.; Wang, F.; Huang, W.; Hao, G.; Yang, G.-F. Auto in Silico Ligand Directing Evolution to Facilitate the Rapid and Efficient Discovery of Drug Lead. iScience 2020, 23, 101179. [CrossRef]

24. Gopalsamy, A.; Shi, M.; Boschelli, D.H.; Williamson, R.; Olland, A.; Hu, Y.; Krishnamurthy, G.; Han, X.; Arndt, A.K.; Guo, B. Discovery of Dibenzo[c,f][2,7]naphthyridines as Potent and Selective 3-Phosphoinositide-Dependent Kinase-1 Inhibitors. J. Med. Chem. 2007, 50, 5547-5549. [CrossRef] [PubMed]

25. Litvinov, V. Advances in the Chemistry of Naphthyridines. Adv. Heterocycl. Chem. 2006, 91, 189-300. [CrossRef]

26. Suzuki, H.; Sakai, N.; Iwahara, R.; Fujiwaka, T.; Satoh, M.; Kakehi, A.; Konakahara, T. Novel Synthesis of 7-Fluoro-8-(trifluoromethyl)- 1H-1,6-naphthyridin-4-one Derivatives: Intermolecular Cyclization of anN-Silyl-1-azaallyl Anion with Perfluoroalkene and Subsequent Intramolecular Skeletal Transformation of the Resulting Pentasubstituted Pyridines. J. Org. Chem. 2007, 72, 5878-5881. [CrossRef]

27. Morgentin, R.; Pasquet, G.; Boutron, P.; Jung, F.; Lamorlette, M.; Maudet, M.; Ple, P. Strategic studies in the syntheses of novel 6,7-substituted quinolones and 7- or 6-substituted 1,6- and 1,7-naphthyridones. Tetrahedron 2008, 64, 2772-2782. [CrossRef]

28. Zeng, L.-F.; Wang, Y.; Kazemi, R.; Xu, S.; Xu, Z.-L.; Sanchez, T.W.; Yang, L.-M.; Debnath, B.; Odde, S.; Xie, H.; et al. Repositioning HIV-1 Integrase Inhibitors for Cancer Therapeutics: 1,6-Naphthyridine-7-carboxamide as a Promising Scaffold with Drug-like Properties. J. Med. Chem. 2012, 55, 9492-9509. [CrossRef]

29. Falardeau, G.; Lachance, H.; St-Pierre, A.; Yannopoulos, C.G.; Drouin, M.; Bédard, J.; Chan, L. Design and synthesis of a potent macrocyclic 1,6-napthyridine anti-human cytomegalovirus (HCMV) inhibitors. Bioorganic Med. Chem. Lett. 2005, 15, 1693-1695. [CrossRef]

30. Wang, Y.; Bullock, W.H.; Chen, L. 1,6-naphthyridine Derivatives as Antidiabetics. WO 03027113A1, 3 April 2003.

31. Sanchez, J.P.; Gogliotti, R.D. The synthesis of a series of 7-amino-1-cyclopropyl-8-fluoro-1,4-dihydro-4-oxo1,6-naphthyridine-3-carboxylic acids as potential antibacterial agents. J. Heterocycl. Chem. 1993, 30, 855-859. [CrossRef]

32. Gündisch, D.; Eibl, C. Nicotinic acetylcholine receptor ligands, a patent review (2006-2011). Expert Opin. Ther. Patents 2011, 21, 1867-1896. [CrossRef] [PubMed]

33. Richard, K. Alpha7 Nicotinic Acetylcholine Receptor Allosteric Modulators, Their Derivatives and Uses Thereof. WO 2010083444A1, 15 January 2009.

34. Dembélé, O.; Montoir, D.; Yvorra, T.; Sérillon, D.; Tonnerre, A.; Duflos, M.; Robert, J.-M.; Bazin, M.-A. Novel approach towards 3,7-disubstituted 1,6-naphthyridin-4(1H)-ones exploiting cross-coupling and SNAr reactions of a dihalogenated compound. Tetrahedron Lett. 2018, 59, 3519-3523. [CrossRef]

35. Kumpina, I.; Isaksson, R.; Sävmarker, J.; Wannberg, J.; Larhed, M. Microwave Promoted Transcarbamylation Reaction of Sulfonylcarbamates under Continuous-Flow Conditions. Org. Process. Res. Dev. 2016, 20, 440-445. [CrossRef]

36. Tabarrini, O.; Stevens, M.; Cecchetti, V.; Sabatini, S.; Dell’Uomo, M.; Manfroni, G.; Palumbo, M.; Pannecouque, C.; De Clercq, E.; Fravolini, A. Structure Modifications of 6-Aminoquinolones with Potent Anti-HIV Activity1. J. Med. Chem. 2004, 47, 5567-5578. [CrossRef] [PubMed]

Sample Availability: Not available.

Publisher's Note: MDPI stays neutral with regard to jurisdictional claims in published maps and institutional affiliations.

(C) 2020 by the authors. Licensee MDPI, Basel, Switzerland. This article is an open access article distributed under the terms and conditions of the Creative Commons Attribution (CC BY) license (http://creativecommons.org/licenses/by/4.0/). 